This report was prepared as an account of work sponsored by an agency of the United States Government. Neither the United States Government nor any agency thereof, nor any of their employees, makes any warranty, express or implied, or assumes any legal liability or responsibility for the accuracy, completeness, or usefulness of any information, apparatus, product, or process disclosed, or represents that its use would not infringe privately owned rights. Reference herein to any specific commercial product, process, or service by trade name, trademark, manufacturer, or otherwise does not necessarily constitute or imply its endorsement, recommendation, or favoring by the United States Government or any agency thereof. The views and opinions of authors expressed herein do not necessarily state or reflect those of the United States Government or any agency thereof.

\title{
A Technique Using a Stellar Spectrographic Plate to Measure Terrestrial Ozone Column Depth
}

\author{
Alec Y. Wong \\ (M.S. Thesis)
}

Manuscript date: August 1995

\section{LAWRENCE LIVERMORE NATIONAL LABORATORY University of California $\bullet$ Livermore, California $\bullet 94551$}

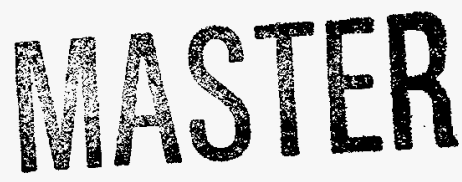




\section{DISCLAIMER}

Portions of this document may be illegible in electronic image products. Images are produced from the best available original document. 
APPROVED FOR THE DEPARTMENT OF PHYSICS

Dr. Patrick Hamill

Dr. Allen Tucker

Dr. Allen Grossman

APPROVED FOR THE UNIVERSITY 


\title{
ABSTRACT \\ A TECHNIQUE USING A STELLAR SPECTROGRAPHIC PLATE TO MEASURE TERRESTRIAL OZONE COLUMN DEPTH
}

\author{
by Alec Y. Wong
}

This thesis examines the feasibility of a technique to extract ozone column depths from photographic stellar spectra in the 5000-7000 Angstrom spectral region. A stellar spectrographic plate is measured to yield the relative intensity distribution of a star's radiation after transmission through the earth's atmosphere. The amount of stellar radiation absorbed by the ozone Chappuis band is proportional to the ozone column depth. The measured column depth is within $10 \%$ the mean monthly value for latitude $36^{\circ} \mathrm{N}$, however the uncertainty is too large to make the measurement useful. This thesis shows that a $10 \%$ improvement to the photographic sensitivity uncertainty can decrease the column depth uncertainty to a level acceptable for climatic study use. This technique offers the possibility of measuring past ozone column depths. 


\section{ACKNOWLEDGMENTS}

I sincerely thank Dr. Allen Grossman for his guidance, collaboration, and discussions for all phases of this work. I also thank Dr. Patrick Hamill for his discussions and advice in writing.

I extend my appreciation to Tony Misch at Lick Observatory for supplying the stellar plate information, Dr. Daniel Popper at UCLA for identifying the spectroscopic lines, Judy Ticehurst at Lawrence Livermore National Laboratory for scanning the plate, and Don Phillion at LLNL for allowing me to use his PDS program.

The work described in this thesis was supported by the Institute for Geophysics and Planetary Physics and the Environmental Programs Directorate, LLNL. 
I. INTRODUCTION

Importance of ozone

Photochemistry

Abundance measurements

II. THEORY

Choosing a star

Absorption and scattering

Chappuis band

III. MEASUREMENT

Stellar plate

Measuring stellar intensity

Atmospheric transmission

Using differential measurements

Applying least squares

IV. ANALYSIS

Uncertainties

Measured column depth

Seasonal average

V. CONCLUSION

Limitations

Improvements

TABLE 1

FIGURES 


\section{LIST OF TABLES}

page

1. Table of relative intensities. 


\section{LIST OF FIGURES}

page

1. Plot of Chappuis absorption band coefficients $\kappa(\lambda)$. 43

2. Sketch of plate calibration by step slit aperture. 44

3. Plot of stellar strip density $D^{*}(\lambda)$. 45

4. Sketch of characteristic curve $D(n, \lambda)$ versus $\log f(n)$. 46

5. Plot of relative stellar intensity $\mathrm{J}_{\text {below }}(\lambda)$. 47

6. Plot of the extra-terrestrial flux $\Phi_{\mathrm{ET}}(\lambda)$ of Zeta Persei. 48

7. Plot of the relative total extinction $a_{\text {total }}(\lambda)$. 49 


\section{INTRODUCTION}

The current concerns about climate change, global warming, and increased ultraviolet surface radiation have necessitated studies of the radiative effects of various trace gases that have been introduced into the atmosphere (WMO, 1994; IPCC, 1990, 1992, 1994). In particular, the atmospheric abundance of ozone plays a pivotal role in the determination of the surface ultraviolet radiation which, in turn, interacts with the major biological systems that exist on the earth's surface (WMO, 1994). Examples of this interaction include the correlation of human skin cancer occurrence with increased surface ultraviolet surface radiation, crop damage effects due to increased surface ultraviolet radiation, and sea surface plankton damage with increased surface ultraviolet radiation (UV-B Monitoring Workshop, 1992).

The primary regulating agent of the surface ultraviolet radiation is the stratospheric ozone layer which provides most of the absorption of the solar ultraviolet radiation entering the atmosphere. Measurements of stratospheric ozone abundance have revealed large areas of depleted ozone abundances over the surface of the earth that have been produced in the last twenty years (WMO, 1994). The largest of these ozone depleted areas is the periodic "ozone hole" found over the south pole (WMO, 1994). The size of the ozone hole has been increasing over the last fifteen years, causing alarm in southern hemisphere countries about increased surface UV radiation effects (WMO, 1994).

One of the major causes of the ozone layer depletion is thought to be the introduction of man-made compounds such as the chlorofluorocarbons (CFCs) which, upon transport into the stratosphere, cause chemical reactions which deplete the ozone layer (WMO, 1994). Validation of the models and measurements relating ozone depletion to the emissions of 
these chemical compounds into the atmosphere on a global and temporal basis is a current and very active area of research (WMO, 1994; UV-B Monitoring Workshop, 1992). An aspect that should be investigated is the possibility of obtaining atmospheric ozone abundances at earlier times in this century before CFC introduction into the atmosphere. These measurements can broaden the time dependent data base of ozone abundance.

Very limited studies have been made (Turco et al., 1981; Angione et al., 1976) to determine the ozone abundances as a function of time for the period 1912-1950 using ozone Chappuis band absorption in the 5000-7000 Angstrom wavelength spectral region. The data base used by Angione et al. was taken from the Smithsonian Institution's Astrophysical Observatory solar constant observing program from 1902-1962 in southern California and northern Chile. These studies showed that the ozone column abundance varied by $20-30 \%$ on time scales ranging from months to decades. The results showed that the mean ozone column abundance values for southern California appeared to be roughly constant between 1912 and 1950 while the northern Chile results seemed to show a marked decrease in the mean ozone column abundance of $\sim 15 \%$ over the same time period.

A method to measure historical ozone abundances is described in this thesis. This method uses a stellar spectra plate from an astronomical observatory to obtain the ground based intensity of a star. The ozone column depth is determined by measuring the amount of stellar intensity absorbed by terrestrial ozone within the Chappuis absorption band. We believe this method can be applied to spectrographic plates taken in the early part of the twentieth century to determine the ozone abundance of the period before the atmospheric release of CFCs. 
The first chapter of this thesis outlines the importance of the ozone layer to earth's environment, ozone photochemistry, a history of previous attempts at measuring ozone abundances, and the advantages of this method for historical ozone column depth measurement. The second chapter outlines the theoretical basis of this measurement method. The third chapter details the measurement procedures of a stellar spectrographic plate. The results of the measurement and an analysis of the uncertainties are presented in chapter four. A discussion of the next steps towards the application of this method is presented in chapter five.

Ozone $\left(\mathrm{O}_{3}\right)$ is a trace atmospheric gas composed of three linearly bonded oxygen atoms in a bent molecular structure. It is a toxic and highly reactive gas in the troposphere. Its concentration peaks at $25 \mathrm{~km}$ above the earth's surface in mid latitudes. $90 \%$ of the ozone in a column depth measurement is found in the stratosphere (UCAR and NOAA, 1992). Stratospheric ozone is a beneficial gas unlike tropospheric ozone which is one of the main constituents of urban smog. Stratospheric ozone is responsible for shielding the earth's surface from ultraviolet radiation.

According to Whitten and Prasad (1985), the existence of an ozone layer led to the evolution of life on the earth's surface. The first life on earth was most likely blue green algae living in the murky oceans depths. The ocean protected the cells from the sun's damaging ultraviolet radiation. These primitive cells converted the abundant carbon dioxide $\left(\mathrm{CO}_{2}\right)$ present in the primordial earth atmosphere to molecular oxygen $\left(\mathrm{O}_{2}\right)$. Approximately a billion years ago, an ozone layer developed from the accumulated molecular oxygen in the atmosphere. The ozone layer absorbed the harmful ultraviolet radiation and allowed life to spread from the ocean onto land. 
The ozone layer shields humans and other surface life from the dangerous ultraviolet radiation of the sun. Near ultraviolet radiation (2500-3800 Angstrom) is categorized into three regions based its health effects : UV-A, UV-B, and UV-C. UV-A radiation ranges from 3200-3800 Angstrom and is generally regarded as benign. It penetrates the atmosphere without suffering significant attenuation (NRC, 1982). UV-B radiation ranges from 2900-3200 Angstrom and is known to cause sunburns, skin cancer, premature aging of the skin, and eye damage. Although most of the sun's UV-B is blocked by the earth's atmosphere, a significant amount reaches the earth's surface (NRC, 1982). UV-C radiation ranges from 2500-2900 Angstrom and is completely blocked by the ozone layer (NRC, 1982). UV-C is lethal to plant and animal life. Speculation regarding the amount of ultraviolet radiation penetrating to the surface of the earth centers around UV-B radiation. A thinner ozone layer would primarily favor increased surface UV-B intensities (NRC, 1982). Several possible outcomes of a thinning ozone layer are increased rates of skin cancer, crop damage, and sea surface plankton damage all due to more surface ultraviolet radiation (UV-B Monitoring Workshop, 1992).

The Hartley and Huggins absorption bands of ozone which span from 2420-3100 Angstrom and 3100-4000 Angstrom respectively (Brasseur and Solomon, 1984) absorb ultraviolet radiation. The Hartley band has its peak absorption within the range of UV-C. All of the sun's UV-C and a portion of the UV-B are prevented from reaching the earth's surface within the wavelength range of the Hartley band (NRC, 1982). The Huggins band begins at the upper wavelength limit of the Hartley band. The Huggins absorption band is approximately 1000 times weaker than the Hartley band (Schachter, 1991). The Huggins band partially absorbs UV-B and UV-A radiation (NRC, 1982).

Ozone photochemistry of the upper atmosphere is a cycle of generation and dissociation 
driven by the absorption of ultraviolet sunlight. The process of ozone creation begins with the photodissociation of molecular oxygen into free atomic oxygen $(\mathrm{O})$ by a far ultraviolet photon hv (wavelength less than 2500 Angstrom):

$$
\mathrm{O}_{2}+\mathrm{hv} \rightarrow \mathrm{O}+\mathrm{O}
$$

The free atomic oxygen reacts with molecular oxygen via a mediator molecule, $\mathrm{M}$, to form ozone:

$$
\mathrm{O}+\mathrm{O}_{2}+\mathrm{M} \rightarrow \mathrm{O}_{3}+\mathrm{M}
$$

The net reaction of ozone generation is :

$$
3 \mathrm{O}_{2}+\mathrm{hv} \rightarrow 2 \mathrm{O}_{3} .
$$

Ozone dissociation begins with an ultraviolet range photon $\mathrm{h} v$ reacting with ozone to form molecular and atomic oxygen :

$$
\mathrm{O}_{3}+\mathrm{hv} \rightarrow \mathrm{O}+\mathrm{O}_{2} \text {. }
$$

The free atomic oxygen bonds with an ozone molecule to produce two oxygen molecules:

$$
\mathrm{O}+\mathrm{O}_{3} \rightarrow \mathrm{O}_{2}+\mathrm{O}_{2} \text {. }
$$

The net reaction of ozone dissociation is :

$$
2 \mathrm{O}_{3}+\mathrm{hv} \rightarrow 3 \mathrm{O}_{2} .
$$

The number of ozone molecules generated and dissociated are equal in a complete cycle. 
However, under non-equilibrium conditions this is not true. For instance, if there is a deficiency of atmospheric ozone, the rate of ozone generation will be greater than dissociation to restore the equilibrium amount (NRC, 1982). The balance of the two competing processes of generation and dissociation regulates the amount of ozone in the atmosphere. At equilibrium, the rate of ozone generation is equal to the natural rate of ozone dissociation, thereby ensuring a constant ozone concentration (NRC, 1982).

The major absorption band of ozone in the 4000-8500 Angstrom region is the Chappuis band (Brasseur and Solomon, 1984). This band is within in the visible spectrum (40008000 Angstrom). Chappuis band absorption is weaker than Hartley band absorption (Griggs, 1968) and it has no role in blocking ultraviolet radiation from reaching the earth's surface. A consequence of Chappuis band absorption of visible light is stratospheric heating (Lacis and Hansen, 1974). Chappuis band absorption is a significant contributor to the stratospheric temperature since this band absorbs within the sun's most intense region (Kyle, 1991). The peaked increase of atmospheric temperature with altitude in the stratosphere is linked to the high concentration of ozone found in the stratosphere absorbing the incident sunlight (Brasseur and Solomon, 1984).

Relatively new chemicals such as CFCs, suspected as the primary catalyst in ozone depletion, were released into the atmosphere in this century. The danger from CFCs is the inert property of the chemical. CFCs do not break down in the troposphere (UCAR and NOAA, 1992). The CFCs in the troposphere are carried into the stratosphere where ultraviolet solar radiation finally photodissociates the molecules and frees the chlorine (CI) atoms (UCAR and NOAA, 1992). The chlorine catalytically reacts with the stratospheric ozone to produce molecular oxygen : 


$$
\begin{gathered}
\mathrm{O}_{3}+\mathrm{Cl} \rightarrow \mathrm{ClO}+\mathrm{O}_{2} \\
\mathrm{ClO}+\mathrm{O}_{3} \rightarrow \mathrm{Cl}+2 \mathrm{O}_{2} .
\end{gathered}
$$

The net catalytic reaction is :

$$
2 \mathrm{O}_{3} \rightarrow 3 \mathrm{O}_{2}
$$

The chlorine atom is still free to react with more ozone molecules. One chlorine atom can destroy a hundred thousand ozone molecules before another atom bonds with the chlorine or the chlorine is transported out of the stratosphere, thereby removing it from the catalytic destruction process of ozone (UCAR and NOAA, 1992). Chlorine upsets the balance of ozone generation and dissociation by accelerating the rate of ozone dissociation while the ozone production rate remains constant (NRC, 1982). CFCs are suspected of contributing to the total stratospheric chlorine burden. There are also natural sources known to release chlorine into the stratosphere. Volcanoes release chlorine in the form of hydrogen chloride, $\mathrm{HCl}$, and biological process from the land and sea release chlorine in the form of methyl chloride, $\mathrm{CH}_{3} \mathrm{Cl}$ (Bower and Ward, 1982).

To investigate the theories regarding anthropogenic sources of ozone depletion, measurements of the ozone abundance before the introduction of CFCs into the atmosphere should be compared with post-CFCs values. CFCs were invented during the 1920's but were not widely used until the 1960's (Bower and Ward, 1982). The Dobson spectrophotometer used to measure ozone column depths was invented in 1924 . Observatories around the world were soon established to perform ozone measurements including : Oxford, England; Table Mountain, California; Helwan, Egypt; Kodai Kanal, India; and Christchurch, New Zealand (Dobson, 1968). The Dobson spectrophotometer 
measures the absorption of solar ultraviolet radiation in the Huggins band to determine atmospheric ozone column depths. All long term monitoring programs in the past have used the Dobson spectrophotometer (Angione et al., 1976). Yet, there are other sources for historical ozone data. The Chappuis absorption band was used by Angione et al. to measure ozone column depth from data obtained by the Smithsonian Institution's Astrophysical Observatory solar constant program from 1902 to 1961 in southern California and northern Chile. Additional ozone column abundance determinations using Chappuis band measurements have been made by Mehra et al. (1986), Shaw (1979), and King and Byrne (1976). The results from these observations constitute part of the global data base for ozone abundance trends. More data regarding past ozone column depths from various geographic locations would extend the data base.

A possible method for determining historical ozone column depths is to analyze old stellar spectrographic plates for the presence of atmospheric ozone absorption bands within the recorded stellar spectra. Astronomical observatories throughout the world archive their old spectrographic plates. The plates could provide an excellent source of well preserved, chronologically and geographically diverse source of ozone abundance data. The plates are permanent records of past atmospheric conditions above the observatory.

The method measures the absorption of stellar radiation through the earth's atmosphere due to the ozone Chappuis band within 5000 to 7000 Angstrom. The Chappuis band is a weak band absorbing approximately $2 \%$ of the total solar flux in mid-latitudes (Angione et al., 1976). The Chappuis band attenuates radiation linearly with respect to the amount of ozone present in the atmosphere unlike the Hartley and Huggins bands which are saturated (Lacis and Hansen, 1974). 
The advantages of measuring the ozone column depth based on the Chappuis absorption band are manyfold. Unlike the Huggins band, Chappuis band absorption coefficients are not temperature dependent (Humphrey and Badger, 1947). The Chappuis absorption coefficients are also not susceptible to pressure broadening (Griggs, 1968). Since the Chappuis band lies in the visible wavelengths, spectrographic plates for visible light astronomy are used. Plates for visible light astronomy constitute all of the pre-1970's spectrographic plates stored in observatory archives, thereby providing a large supply of plates for measurement.

The spectrographic plate offers advantages over traditional detectors. The plate allows a continuous sampling of the Chappuis band's broad absorption spectrum using approximately 2600 data points spanning 2000 Angstroms. In comparison, the Dobson spectrometer uses data from 10 wavelengths to determine the ozone column depth (Whitten and Prasad, 1985). Other atmospheric features that may corrupt the desired ozone band absorption can also be seen on the spectrograph to avoid interference in the final calculation of ozone column depth. 


\section{THEORY}

The method described in this thesis obtains a terrestrial ozone column depth measurement from starlight transmission through the earth's atmosphere. A spectrographic plate of a star is chosen. The source star must be bright enough so that the spectrographic plate signal to noise ratio is acceptable. The star must be relatively clean of stellar spectral lines such that the primary absorption features recorded on the spectrographic plate would be of terrestrial origin. If extra-terrestrial intensity (stellar intensity above the earth's atmosphere) measurements of the star cannot be found in the literature, the intensity can be calculated by assuming the star radiates as a perfect blackbody. Lastly, the star should have been observed through an acceptable airmass such that the path of the stellar radiation through the earth's atmosphere is kept to a minimum.

The spectral class of stars chosen in this study is type B of luminosity I. B type stars range in temperature from $12000-28000 \mathrm{~K}$ and appear blue in color. The spectra of B stars are relatively featureless, unlike cooler stars that have spectra heavily dominated by absorption lines of metals (Aller, 1963). Luminosity I designates the star as an ordinary supergiant. B stars of luminosity I were chosen because they best fit the above conditions and spectrographic plates of these bright stars are relatively abundant.

The spectrographic plate consists of a strip of glass with a layer of photographic emulsion. The photographic emulsion is a mixture of silver halide crystals held in a gelatin suspension. When photons are absorbed by a silver halide crystal, the crystal reduces to silver atoms which turn black upon development (Morton, 1984). By measuring the density of these blackened crystals a determination of the terrestrial intensity of the starlight can be made. 
The extra-terrestrial intensity distribution of the chosen star is found in published measurements of stellar intensities. Selected stellar intensities are measured for use as photometric standards in star catalogs. The extra-terrestrial energy distribution of the star used in this thesis is taken from spectrophotometric measurements by Glushneva et al. (1992). If the extra-terrestrial intensity data cannot be found in the literature, the intensity distribution can be determined by assuming the star radiates as a blackbody. The extraterrestrial intensity distribution can be calculated using Planck's blackbody radiation law and the effective temperature of the star based on the spectral type.

The stellar radiation is unattenuated as it traverses through the interstellar medium save for absorption by interstellar gases and particles which contribute very weak and narrow absorption bands (diffuse interstellar bands) observed on the spectrographic plate (Herbig, 1975). A telescope on the ground directed towards the source star only detects the starlight that reaches the aperture. Any stellar light that is absorbed by earth's atmospheric gases or scattered away from the line of sight of the star by terrestrial dust results in less direct starlight incident upon the telescope. The ozone measurement method described in this thesis accounts for the attenuation of stellar light from atmospheric scattering and absorption by terrestrial particles and gases.

The two principle scattering mechanisms are aerosol and Rayleigh scattering. Aerosol scattering is caused by light scattering off dust particles in the atmosphere. Rayleigh scattering is due to light scattering off gas molecules. Both aerosol and Rayleigh scattering are wavelength dependent and attenuate continuously across the spectrum. Aerosols attenuate radiation approximately linearly with respect to wavelength, whereas Rayleigh scattering varies inversely as the fourth power of wavelength (Mehra et al., 1986). 
Absorption is caused by radiative excitation of gas molecules to higher energy states, either to a higher vibrational/rotational mode or a higher electronic transition state as in the Huggins and Hartley bands (Whitten and Prasad, 1985). The original incident photon is absorbed by the molecule and is not re-radiated as in the case of pure scattering (Buglia, 1986). Chappuis band absorption is a vibrational excitation mode (Kyle, 1991). This absorption band has peak radiation attenuation in the visible range (Griggs, 1968). The shape of the Chappuis band is distinguished by two peaks at 5800 and 6000 Angstrom and a dip at 5900 Angstrom [Figure 1]. Other atmospheric gases also have distinct absorption signatures within the Chappuis band's wavelength range (Angione and Roosen, 1981). Molecular oxygen and water do not absorb radiation as wide ranging as the Chappuis band but their strong absorption features in the visible spectrum dwarf the much weaker Chappuis band absorption.

The direct radiation from the star incident upon the telescope is collected by the lens and focused onto a spectrograph. The stellar spectrum is recorded on a photographic plate. The plate becomes a permanent record of the stellar emission and earth's atmospheric transmission spectra. The densities of the exposed spectrographic plate emulsion are measured and interpreted as the energy distribution of the star after transmission through the earth's atmosphere (terrestrial intensity).

The measured total atmospheric extinction of stellar radiation is the difference between the star's (logarithmic) extra-terrestrial and terrestrial intensities. The total atmospheric extinction can be expressed as the sum of separate extinctions due to aerosol and Rayleigh scattering and absorption from ozone, oxygen, water and other gases (Walker, 1992). The ozone column abundance, Rayleigh scattering, and aerosol scattering are measured after removing the extinctions due to absorption by oxygen and water vapor from the total atmospheric extinction. 


\section{MEASUREMENT}

Measurement of ozone column depth begins with obtaining a stellar spectrographic plate. The plate used for this method was exposed for 16 minutes at Lick Observatory on the 120 inch Shane reflecting telescope starting at 10:24 UT September 10/11, 1963. The star chosen for that night was Zeta Persei (HD 24398, declination $+31^{\circ} 35^{\prime}$ ). Zeta Persei is a BI spectral class star with an effective temperature on the order of $20000 \mathrm{~K}$ (Theodossious, 1985). The plate was originally intended to be used in a study of diffuse interstellar bands. The stellar light, the light from an intensity calibration lamp, and a wavelength calibration lamp were incident on a Coude spectrograph with a dispersion of 8.2 Angstroms per millimeter using a grating of 600 groove per millimeter. A photographic plate recorded each of the spectra.

The photographic plate on which the spectra are recorded is a Kodak 103a-F emulsion designed for visible light spectroscopy. There are eight darkened strips on the plate corresponding to three functions of the spectrograph: wavelength calibration, stellar intensity, and intensity calibration. The emission spectrum of an NeI lamp is recorded on the plate for use as a wavelength standard to compare its known emission spectrum to the wavelength positions of the energy calibration and stellar spectra. The six intensity calibration strips on the plate generated by a step slit aperture and a calibration lamp determine the relative intensities of the stellar strip [Figure 2].

The step slit aperture consists of slits of varying widths, $n$, which govern the solid angle, $\Omega(n)$, subtended by the aperture-plate arrangement. Let width $n$ also identify the corresponding intensity calibration strip. The fluxes incident on the plate, $\Phi_{\text {plate }}(n, \lambda)$, are proportional to the solid angles $\Omega(n)$ and the intensity of the lamp $I_{0}(\lambda)$. At each 
calibration strip the flux incident on the plate is :

$$
\Phi_{\text {plate }}(\mathrm{n}, \lambda)=\mathrm{I}_{\mathrm{o}}(\lambda) \cdot \Omega(\mathrm{n})
$$

The density of calibration strip $n, D(n, \lambda)$, is proportional the flux incident on the plate :

$$
\mathrm{D}(\mathrm{n}, \lambda) \propto \Phi_{\text {plate }}(\mathrm{n}, \lambda)=\mathrm{I}_{\mathrm{o}}(\lambda) \cdot \Omega(\mathrm{n})
$$

However, the above relationship can be rewritten as :

$$
\mathrm{D}(\mathrm{n}, \lambda) \propto \Phi_{\text {plate }}(\mathrm{n}, \lambda)=\mathrm{I}(\mathrm{n}, \lambda) \cdot \Omega
$$

The above equation assumes the aperture solid angle $\Omega$ is constant for the set of calibration strips while the apparent intensities incident on the strips, $I(n, \lambda)$, vary with respect to $\mathrm{n}$. The interpretation of the six calibration strips on the plate is a photographic record of the emulsion response to various lamp intensities at various wavelengths.

Since the intensity distribution of the calibration lamp $I_{0}(\lambda)$ is unknown for this plate, the step-slit aperture imposes a relative intensity scale in the measurement of calibration strip intensities. The flux from the calibration lamp is :

$$
\Phi_{0}(\lambda)=\mathrm{I}_{0}(\lambda) \cdot \Omega
$$

The ratios of intensities (i.e. fluxes) with respect to the slits are denoted as $f(n)$ :

$$
\mathrm{f}(\mathrm{n})=\frac{\Phi_{\text {plate }}(\mathrm{n}, \lambda)}{\Phi_{\mathrm{o}}(\lambda)}=\frac{\mathrm{I}(\mathrm{n}, \lambda) \cdot \Omega}{\mathrm{I}_{\mathrm{o}}(\lambda) \cdot \Omega}=\frac{\mathrm{I}(\mathrm{n}, \lambda)}{\mathrm{I}_{\mathrm{o}}(\lambda)}
$$

The intensity ratios $f(n)$ obtained from Lick Observatory Technical Reports No. 18 
(Soderblom, 1976) are listed in Table 1.

The density of emulsion strip $\mathrm{n}$ is dependent on the energy per area incident on the plate. The energy per area incident on the plate, $\varepsilon(n, \lambda)$, is a function of the flux $\Phi(n, \lambda)$ and the amount of time $t$ exposing the plate :

$$
\varepsilon(\mathrm{n}, \lambda)=\Phi(\mathrm{n}, \lambda) \cdot \mathrm{t} .
$$

Expand the flux in terms of intensity and solid angle :

$$
\varepsilon(\mathrm{n}, \lambda)=\mathrm{I}(\mathrm{n}, \lambda) \cdot \Omega \cdot \mathrm{t} .
$$

Take the logarithm of the energy density $\varepsilon(n, \lambda)$ :

$$
\log \varepsilon(\mathrm{n}, \lambda)=\log \mathrm{I}(\mathrm{n}, \lambda)+(\log \Omega+\log \mathrm{t})=\log \mathrm{I}(\mathrm{n}, \lambda)+\text { const } .
$$

The energy density deposited on the plate (i.e. density of darkened emulsion) varies only with intensity $I(n, \lambda)$ since the solid angle and exposure time are constant for each calibration strip and are not functions of wavelength.

The emulsion response to light energy is characterized by the type of emulsion and development process. Each type of emulsion has a unique energy density threshold which produces a given emulsion density. This threshold energy density, $\Phi_{\text {threshold }}(\lambda) t$, is written as the emulsion spectral sensitivity e $(\lambda)$ (James, 1977) :

$$
\mathrm{e}(\lambda)=-\log \Phi_{\text {threshold }}(\lambda)+\text { const }
$$

The sensitivity e $(\lambda)$ used in this measurement is from Kodak (1994). Each astronomical 
observatory has its own in-laboratory method of processing a photographic plate before and after exposure to customize the plates for optimizing detection of dim stars. The astronomer may hyper-sensitize the plates, pre-expose, bake, or chemically treat them. These techniques have the overall effect of increasing the sensitivity e $(\lambda)$ of the plate. The plate used in this study was not treated for sensitivity enhancement. Contrast $\gamma(\lambda)$ is defined as the rate of emulsion darkening with respect to the logarithm of exposed light intensity. The type of developer used and development time determine the contrast of the plate.

The scanning facility at Lawrence Livermore National Laboratory measures the densities on the spectrographic plate using a densitometer. Densitometry consists of transmitting light from a lamp of known intensity through an area of the developed plate located at position $\mathrm{x}$ and measuring the attenuated intensity on the other side with a photocell. The area measured is adjusted by the width of the densitometer aperture which determines the wavelength resolution of the scan. The attenuation of the lamp intensity is due to the density of the darkened emulsion blocking out the light from the lamp as the light traverses the plate. The plate's transmission of light, $T_{\text {plate }}(x)$, is the ratio of the intensity measured by the photocell versus the intensity of the lamp.

The density of the darkened emulsion, $\mathrm{D}(\mathrm{x})$, is proportional to the logarithm of the plate transmission (Levi, 1980) :

$$
\mathrm{D}(\mathrm{x}) \propto-\log \mathrm{T}_{\text {plate }}(\mathrm{x})
$$

The emulsion density of each strip on the plate is measured. The densities of the wavelength calibration strip correspond to emission lines of NeI, thereby fixing 
wavelength positions for the calibration and stellar strips by converting the dispersion position $\mathrm{x}$ to the corresponding wavelength $\lambda$ [Figure 3] :

$$
\mathrm{D}(\lambda) \propto-\log \mathrm{T}_{\text {plate }}(\lambda)
$$

The wavelength and density resolutions of the scan are 1 Angstrom and 0.00001 density respectively, using a densitometer aperture width of $100 \mu \mathrm{m}$. Designate the densities of calibration strip $n$ as $D(n, \lambda)$ and the densities of the stellar strip as $D *(\lambda)$.

Characteristic curves of the spectrographic plate are constructed by plotting the calibration strip densities $D(n, \lambda)$ with respect to the logarithm of relative intensities $f(n)$ for each wavelength $\lambda$ [Figure 4]. There are three regions of the characteristic curve : toe, linear, and shoulder. The toe region is the response of the emulsion to low intensities. Many irregular densities unrelated to the incident intensities can be seen in this region. These spurious densities, called fog, are caused by the nonuniform distribution of the silver halide crystals in the emulsion layer. Photometric measurements are usually made above the fog level to avoid noisy readings.

The density is linearly proportional to the intensity of light in the linear region of the characteristic curve. This is the desired response of the emulsion. The contrast $\gamma^{\prime}(\lambda)$ is the slope of the linear region of the characteristic curve. The shoulder region is caused by the saturation of exposed emulsion crystals. The linear increase of density in relation to intensity ceases as the number of unexposed crystals diminish. The exposed crystals begin to block out the incident light from reaching the unexposed crystals.

The logarithm of the minimum incident relative intensity required to develop an emulsion 
density of a pre-determined value is defined as $e^{\prime}(\lambda)$. Typically a density of 0.6 is chosen. The justification for this definition of $\mathrm{e}^{\prime}(\lambda)$ is to ensure that photometric measurements of plate sensitivity are made in the linear region of the characteristic curve.

The intensity measured from the characteristic curve is on a relative scale. Recall the definition of relative intensity $f(n)$ from Equation [15]. Write $f(n)$ as the logarithmic relative intensity :

$$
\begin{aligned}
\log f(n) & =\log \Phi_{\text {plate }}(n, \lambda)-\log \Phi_{o}(\lambda) \\
& =\log I(n, \lambda)-\log I_{o}(\lambda)
\end{aligned}
$$

Similarly, the logarithmic relative intensity at 0.6 density, $e^{\prime}(\lambda)$, is :

$$
\begin{aligned}
\mathrm{e}^{\prime}(\lambda)= & \log \mathrm{f}_{\mathrm{D}=0.6} \\
& =\log \Phi_{\text {threshold }}(\lambda)-\log \Phi_{\mathrm{o}}(\lambda) \\
& =-\mathrm{e}(\lambda)-\left(\log \mathrm{I}_{\mathrm{o}}(\lambda)+\log \Omega\right)+\mathrm{const} \\
& =-\mathrm{e}(\lambda)-\log \mathrm{I}_{\mathrm{o}}(\lambda)+\mathrm{const}
\end{aligned}
$$

The useful part of the characteristic curve is the linear region. The equation describing the linear region of the calibration strips is :

$$
D(n, \lambda)-0.6=\gamma^{\prime}(\lambda)\left[\log f(n)-e^{\prime}(\lambda)\right]
$$

Rewrite the above expression by substituting in the definition of $\log f(\mathrm{n})$ from Equation [22] : 


$$
D(n, \lambda)-0.6=\gamma^{\prime}(\lambda)\left[\log I(n, \lambda)-\left\{\log I_{o}(\lambda)+e^{\prime}(\lambda)\right\}\right]
$$

Replace $\log I_{o}(\lambda)+e^{\prime}(\lambda)$ with $-\mathrm{e}(\lambda)+$ const as stated by Equation [22] :

$$
D(n, \lambda)-0.6=\gamma^{\prime}(\lambda)[\log I(n, \lambda)+e(\lambda)+\text { const }]
$$

Solve for $\log I(n, \lambda)$ :

$$
\log \mathrm{I}(\mathrm{n}, \lambda)=\frac{\mathrm{D}(\mathrm{n}, \lambda)-0.6}{\gamma^{\prime}(\lambda)}-\mathrm{e}(\lambda)+\mathrm{const}
$$

$\log \mathrm{I}(\mathrm{n}, \lambda)$ is the relative intensity incident on calibration strip $\mathrm{n}$ of density $\mathrm{D}(\mathrm{n}, \lambda)$. Replace $D(n, \lambda)$ with stellar strip density $D^{*}(\lambda)$ to calculate the relative incident stellar intensity at earth's surface, $I^{*}(\lambda)$ :

$$
\log I^{*}(\lambda)=\frac{D^{*}(\lambda)-0.6}{\gamma^{\prime}(\lambda)}-\mathrm{e}(\lambda)+\text { const }
$$

It can be demonstrated that the spectrographic instrument response, $\zeta(\lambda)$, the solid angle subtended by the telescope, $\Omega^{\prime}$, and the stellar radiation exposure time, $t^{\prime}$, do not contribute to the relative stellar intensity calculation. Density $D^{*}(\lambda)$ is a function of the stellar energy density incident on the plate, $J_{\text {below }}(\lambda) \Omega^{\prime} t^{\prime} \zeta(\lambda)$, where $J_{\text {below }}(\lambda)$ is the absolute stellar intensity at the earth's surface. Instrument response in the calculation of stellar intensity is avoided by defining $g^{*}(\lambda)$ as the ratio of the incident stellar energy density versus the incident calibration lamp energy density, $I_{0}(\lambda) \Omega t \zeta(\lambda)$ (Storer, 1928):

$$
g^{*}(\lambda)=\frac{J_{\text {below }}(\lambda) \cdot \Omega^{\prime} \cdot t^{\prime} \cdot \zeta(\lambda)}{I_{o}(\lambda) \cdot \Omega \cdot t \cdot \zeta(\lambda)}=\frac{J_{\text {below }}(\lambda)}{I_{o}(\lambda)} \cdot \frac{\Omega^{\prime} \cdot t^{\prime}}{\Omega \cdot t}
$$


The ratio of stellar intensity versus lamp intensity is :

$$
f^{*}(\lambda)=\frac{J_{\text {below }}(\lambda)}{I_{0}(\lambda)}
$$

Rewrite Equation [29] as :

$$
g^{*}(\lambda)=f^{*}(\lambda) \cdot \text { const } .
$$

where const $=\frac{\Omega^{\prime} \cdot \mathrm{t}^{\prime}}{\Omega \cdot \mathrm{t}}$

Using $g^{*}(\lambda)$ instead of $f(n)$, the form of the characteristic equation (Equation [24]) for the stellar intensity strip is :

$$
D^{*}(\lambda)-0.6=\gamma^{\prime}(\lambda)\left[\log g^{*}(\lambda)-e^{\prime}(\lambda)\right]
$$

Replace $g^{*}(\lambda)$ with its expansion in terms of intensities (Equation [30] and [29]) and solve for $\log \mathrm{J}_{\text {below }}(\lambda)$ :

$$
\log \mathrm{J}_{\text {below }}(\lambda)=\frac{D^{*}(\lambda)-0.6}{\gamma^{\prime}(\lambda)}-\mathrm{e}(\lambda)+\text { const }
$$

The calculated intensity is a constant apart from the "true" stellar intensity $\log \mathrm{J}_{\text {below }}(\lambda)$. Therefore the experimental values, $D^{*}(\lambda), \gamma^{\prime}(\lambda)$, and $e(\lambda)$, determine the relative stellar intensity at the earth's surface without interference from instrument spectral response, telescope aperture size, or exposure time [Figure 5]. 
Atmospheric transmission $T_{\text {atmosphere }}(\lambda)$ is determined by the amount of stellar light that remains unabsorbed and unscattered as it traverses through the atmosphere. The stellar intensities $\mathrm{J}_{\text {above }}(\lambda)$ and $\mathrm{J}_{\text {below }}(\lambda)$ are measured above and below the atmosphere respectively. $\mathrm{J}_{\text {above }}(\lambda)$ is obtained from Glushneva's (1992) extra-terrestrial flux $\Phi_{\mathrm{ET}}(\lambda)$ [Figure 6]:

$$
\log \Phi_{\mathrm{ET}}(\lambda)=\log \left(\mathrm{J}_{\mathrm{above}}(\lambda) \cdot \Omega\right)=\log \mathrm{J}_{\mathrm{above}}(\lambda)+\log \Omega=\log \mathrm{J}_{\mathrm{above}}(\lambda)+\text { const } .
$$

The transmission $T_{\text {atmosphere }}(\lambda)$ is the ratio of the intensities below and above the atmosphere :

$$
\mathrm{T}_{\text {atmosphere }}(\lambda)=\frac{\mathrm{J}_{\text {below }}(\lambda)}{\mathrm{J}_{\text {above }}(\lambda)} .
$$

Another way to define transmission $T_{\text {atmosphere }}(\lambda)$ is (Angione and Roosen, 1983):

$$
\mathrm{T}_{\text {atmosphere }}(\lambda)=10^{-\mathrm{a}_{\text {total }}(\lambda)}
$$

$\mathrm{a}_{\text {total }}(\lambda)$ is the total atmospheric extinction of the stellar radiation. Take the logarithm of the transmission :

$$
\mathrm{a}_{\text {total }}(\lambda)=\log \mathrm{J}_{\text {above }}(\lambda)-\log \mathrm{J}_{\text {below }}(\lambda)
$$

The extinction is the difference of the logarithms of the stellar intensities above and below the atmosphere. Subtract $\log \Phi_{\mathrm{ET}}(\lambda)$ (Equation [33]) and $\log \mathrm{J}_{\text {below }}(\lambda)$ (Equation [32]) to solve for $a_{\text {total }}(\lambda)$ :

$$
\log \Phi_{\mathrm{ET}}(\lambda)-\log \mathrm{J}_{\text {below }}(\lambda)=\log \mathrm{J}_{\text {above }}(\lambda)-\log \mathrm{J}_{\text {below }}(\lambda)+\log \Omega=\mathrm{a}_{\text {total }}(\lambda)+\text { const . [37] }
$$


The resulting total extinction includes a constant term from the solid angle [Figure 7].

The spectral region of the total extinction $a_{\text {total }}(\lambda)$ ranges from $5000-7000$ Angstrom. Gases and particles other than ozone also contribute to the total extinction in this region. The total extinction is the sum of extinctions from Rayleigh scattering, $a_{\text {Rayleigh }}(\lambda)$; aerosol scattering, a aerosol $(\lambda)$; molecular oxygen absorption, $\mathrm{a}_{\mathrm{O}_{2}}(\lambda)$; water vapor absorption, $\mathrm{a}_{\mathrm{H}_{2} \mathrm{O}}(\lambda)$; and ozone absorption, $\mathrm{a}_{\text {ozone }}(\lambda)$ (Walker, 1992):

$$
a_{\text {total }}(\lambda)=a_{\text {Rayleigh }}(\lambda)+a_{\text {aerosol }}(\lambda)+a_{\mathrm{O}_{2}}(\lambda)+a_{H_{2} \mathrm{O}}(\lambda)+a_{\text {ozone }}(\lambda)
$$

It is necessary to identify the atmospheric features across the 5000-7000 Angstrom spectral range in order to find a region with minimal absorption due to molecular oxygen and water vapor (Angione and Roosen, 1981). Water and molecular oxygen bands are identified and located using an atlas of the solar spectra from 2935 to 8770 Angstrom (Moore et al., 1966), uncorrected for terrestrial absorption. Avoiding these bands yields a "clear window" of wavelengths $\lambda$ ' as a subset of all wavelengths $\lambda$ in the 5000-7000 Angstrom spectral range :

$$
\begin{gathered}
\mathrm{a}_{\mathrm{O}_{2}}\left(\lambda^{\prime}\right)=0 \\
\mathrm{a}_{\mathrm{H}_{2} \mathrm{O}}\left(\lambda^{\prime}\right)=0 \\
a_{\text {total }}\left(\lambda^{\prime}\right)=\mathrm{a}_{\text {Rayleigh }}\left(\lambda^{\prime}\right)+\mathrm{a}_{\text {aerosol }}\left(\lambda^{\prime}\right)+\mathrm{a}_{\text {ozone }}\left(\lambda^{\prime}\right) .
\end{gathered}
$$

The clear window used in this measurement is from 5470-5870 Angstrom. Only Rayleigh scattering, aerosol scattering, and ozone absorption contribute to the extinction in the clear window (King and Byrne, 1976). 
The extinction data is smoothed to remove high frequency noise. The noise consists of strong absorption lines of terrestrial and stellar origins such as $\mathrm{Na} \mathrm{D}$ lines and $\mathrm{H} \alpha$ line respectively (The wavelength resolution of Glushneva's data is not fine enough to remove stellar lines.) The noise is removed using boxcar averaging of the data points. The width of the boxcar averaging is chosen with an eye on minimizing the noise while preserving the overall shape of the extinction curve. The Chappuis band is not affected by the data smoothing. The Chappuis band has broad absorption features spanning 2000 Angstroms while the noise is a very narrow feature spanning 10 Angstroms at maximum. The smoothed extinction data is composed of Rayleigh scattering, aerosol scattering, and ozone absorption which attenuate continuously across the spectrum.

A commonly seen effect of Rayleigh scattering is the color of the sky (Houghton, 1985). Rayleigh scattering relies upon gas molecules smaller than the wavelength of visible light. Sunlight scatters off the gas molecules as it travels through the atmosphere. Blue light is strongly scattered from the direct path whereas red light is weakly scattered. The strong scattering of blue sunlight creates the diffuse blue color of the atmosphere during the day while the weak scattering of red sunlight is seen at dusk as the reddening of the horizon. Rayleigh scattering extinguishes direct radiation as the inverse of the fourth power of wavelength (Mehra et al., 1986). The Rayleigh scattering extinction is :

$$
a_{\text {Rayleigh }}(\lambda)=\frac{\rho}{\lambda^{4}}
$$

$\rho$ is the Rayleigh scattering coefficient. Attenuation of direct light due to Rayleigh scattering is heavily weighted towards shorter wavelengths. Rayleigh scattering remains relatively constant (King and Byrne, 1976). 
Aerosol scattering, also known as Mie scattering, can change daily (Houghton, 1985). Small dust particles are responsible for this type of scattering. Aerosol scattering can be minimized since most observatories are located high enough to avoid the majority of anthropogenic aerosols from cities and industries and astronomers can pick observation nights of optimal clarity (Hayes, 1970). Aerosol scattering extinguishes direct radiation approximately linearly with respect to wavelength. Aerosol scattering extinction is :

$$
\mathrm{a}_{\text {aerosol }}(\lambda)=\alpha \cdot \lambda+\text { const }
$$

$\alpha$ is the aerosol scattering coefficient. The attenuation from aerosols is weighted slightly towards shorter wavelengths but this weighting is so small that many measurement schemes, such as the Dobson spectrophotometer, consider aerosol scattering as a grey attenuator, i.e. constant with wavelength (King and Byrne, 1976).

The absorption band of ozone in the visible spectrum is the Chappuis band. Absorption coefficients were obtained from Inn and Tanaka (1953). Chappuis absorption is nearly independent of temperature and pressure making it a good band for measuring ozone since conditions in the stratosphere rarely emulate the laboratory (Griggs, 1968). The absorption coefficients $\kappa(\lambda)$ are given in terms of extinction per atmosphere-cm (atm-cm). Atm-cm is the unit used to quantify the overhead ozone abundance which is defined as a column depth, $\eta$. The column is an imaginary vertical column of atmospheric ozone of one square centimeter cross sectional area extending from the surface of the earth to space. The height of this column is called the column depth, measured in atm-cm, if the entire column of ozone is compressed to one atmospheric pressure. Multiply the column depth given in units of atm-cm by 1000 to convert from atm-cm to Dobson units (DU) (Hamill and Toon, 
1992). The extinction due to the Chappuis band is given as:

$$
\mathrm{a}_{\text {ozone }}(\lambda)=\eta^{\prime} \cdot \kappa(\lambda)
$$

The ozone absorption column depth is calculated by applying the method of least squares (King and Byrne, 1976) to the total extinction data. A differential scheme is used to remove the constants involved in the measurements. The differential extinction, $\Delta \mathrm{a}_{\mathrm{xyz}}^{\mathbf{i}}$, is the difference of extinctions $a_{x y z}\left(\lambda_{n}\right)$ and $a_{x y z}\left(\lambda_{m}\right)$ (xyz is the label of the extinction mechanism). $\lambda_{\mathrm{n}}$ and $\lambda_{\mathrm{m}}$ denote unique wavelengths and $\mathrm{i}$ identifies the unique pair of $\lambda_{\mathrm{n}}$ and $\lambda_{\mathrm{m}}$. The differential extinction for Rayleigh scattering, $\Delta \mathrm{a}_{\text {Rayleigh }}^{\mathrm{i}}$, is :

$$
\Delta a_{\text {Rayleigh }}^{i}=a_{\text {Rayleigh }}\left(\lambda_{n}\right)-a_{\text {Rayleigh }}\left(\lambda_{m}\right)=\rho\left(\lambda_{n}^{-4}-\lambda_{m}^{-4}\right)=\rho\left(\text { Rayleigh }^{i}\right),
$$

where $\lambda_{\mathrm{n}}^{-4}-\lambda_{\mathrm{m}}^{-4} \equiv$ Rayleigh $\mathrm{h}^{\mathrm{i}}$

The differential extinction for aerosol scattering, $\Delta \mathrm{a}^{\mathrm{i}}{ }_{\text {aerosol }}$, is :

$$
\Delta \mathrm{a}_{\text {aerosol }}^{\mathrm{i}}=\mathrm{a}_{\text {aerosol }}\left(\lambda_{\mathrm{n}}\right)-\mathrm{a}_{\text {aerosol }}\left(\lambda_{\mathrm{m}}\right)=\alpha\left(\lambda_{\mathrm{n}}-\lambda_{\mathrm{m}}\right)=\alpha\left(\text { aerosol }^{\mathrm{i}}\right),
$$

where $\lambda_{\mathrm{n}}-\lambda_{\mathrm{m}} \equiv \operatorname{aerosol}^{\mathrm{i}}$.

The differential extinction for ozone absorption in the Chappuis band, $\Delta \mathrm{a}^{\mathrm{i}}{ }_{\mathrm{ozone}}$, is :

$$
\Delta \mathrm{a}_{\text {ozone }}^{\mathrm{i}}=\mathrm{a}_{\text {aerosol }}\left(\lambda_{\mathrm{n}}\right)-\mathrm{a}_{\text {aerosol }}\left(\lambda_{\mathrm{m}}\right)=\eta^{\prime}\left[\kappa\left(\lambda_{\mathrm{n}}\right)-\kappa\left(\lambda_{\mathrm{m}}\right)\right]=\eta^{\prime}\left(\text { Chap }{ }^{\mathrm{i}}\right),
$$

where $\kappa\left(\lambda_{n}\right)-\kappa\left(\lambda_{m}\right) \equiv \operatorname{Chap}^{\mathrm{i}}$

The experimental differential total extinction, $\Delta \mathrm{a}_{\text {total }}^{\mathrm{i}}$, is the difference of a pair of total 
extinctions, $a_{\text {total }}\left(\lambda_{n}\right)$ and $a_{\text {total }}\left(\lambda_{m}\right)$, from Equation [37] :

$$
\begin{aligned}
\Delta \mathrm{a}_{\text {total }}^{\mathrm{i}} & =\mathrm{a}_{\text {total }}\left(\lambda_{\mathrm{n}}\right)-\mathrm{a}_{\text {total }}\left(\lambda_{\mathrm{ma}}\right) \\
& =\log \Phi_{\mathrm{ET}}\left(\lambda_{\mathrm{n}}\right)-\log \mathrm{J}_{\text {below }}\left(\lambda_{\mathrm{n}}\right)-\log \Phi_{\mathrm{ET}}\left(\lambda_{\mathrm{m}}\right)+\log \mathrm{J}_{\text {below }}\left(\lambda_{\mathrm{m}}\right) .
\end{aligned}
$$

The theoretical differential total extinction, $\Delta \mathrm{a}^{\mathrm{a}^{\mathrm{i}}}$ total , is the sum of the Rayleigh scattering, aerosol scattering, and ozone absorption differential extinctions :

$$
\begin{aligned}
& \Delta \mathrm{a}^{,{ }_{\text {total }}}=\Delta \mathrm{a}_{\text {Rayleigh }}^{\mathrm{i}}+\Delta \mathrm{a}_{\text {aerosol }}^{\mathrm{i}}+\Delta \mathrm{a}_{\text {ozone }}^{\mathrm{i}} \\
& =\rho\left(\lambda_{n}^{-4}-\lambda_{m}^{-4}\right)+\alpha\left(\lambda_{n}-\lambda_{m}\right)+\eta^{\prime}\left[\kappa\left(\lambda_{n}\right)-\kappa\left(\lambda_{m}\right)\right] \\
& =\rho\left(\text { Rayleigh }^{\mathrm{i}}\right)+\alpha\left(\text { aerosol }^{\mathrm{i}}\right)+\eta^{\prime}\left(\mathrm{Chap}^{\mathrm{i}}\right)
\end{aligned}
$$

Combine the two forms of the differential total extinction:

$$
\Delta \mathrm{a}_{\text {total }}^{\mathrm{i}} \cong \Delta \mathrm{a}^{\mathrm{i}}{ }_{\text {total }}
$$

The experimental and theoretical differential extinctions are assumed to be equivalent. The above equation is a restatement of the Bouger-Lambert law (Johnson, 1954) :

$$
\Delta \mathrm{a}_{\text {total }}^{\mathrm{i}}=\rho\left(\text { Rayleigh }^{\mathrm{i}}\right)+\alpha\left(\text { aerosol }^{\mathrm{i}}\right)+\eta^{\prime}\left(\text { Chap }^{\mathrm{i}}\right)
$$

Given $\mathrm{N}$ wavelength data points, the number of possible combinations $\mathrm{K}$ of unique pairs of wavelengths $\lambda_{n}$ and $\lambda_{m}$ is :

$$
\mathrm{K}=\left(\begin{array}{c}
\mathrm{N} \\
2
\end{array}\right)=\frac{\mathrm{N} !}{(\mathrm{N}-2) ! 2}
$$


Using the method of least squares (King and Byrne, 1976), the variance $\chi^{2}$ of the Equation [51] is expressed as :

$$
\chi^{2}=\sum_{i}^{K}\left[\Delta a_{\text {total }}^{i}-\rho\left(\text { Rayleigh }^{i}\right)-\alpha\left(\text { aerosol }^{i}\right)-\eta^{\prime}\left(\text { Chap }^{i}\right)\right]^{2} .
$$

Take partial derivatives of $\chi^{2}$ with respect to $\rho, \alpha$, and $\eta^{\prime}$ to minimize the variance :

$$
\begin{aligned}
& \frac{\partial}{\partial \rho} \chi^{2}=-2 \cdot \sum_{i}^{K}\left[\Delta a_{\text {total }}^{i}-\rho\left(\text { Rayleigh }^{i}\right)-\alpha\left(\text { aerosol }^{i}\right)-\eta^{\prime}\left(\text { Chap }^{\mathrm{i}}\right)\right] \cdot \text { Rayleigh }^{\mathrm{i}} \\
& \frac{\partial}{\partial \alpha} \chi^{2}=-2 \cdot \sum_{i}^{\mathrm{K}}\left[\Delta \mathrm{a}_{\text {total }}^{\mathrm{i}}-\rho\left(\text { Rayleigh }^{\mathrm{i}}\right)-\alpha\left(\text { aerosol }^{\mathrm{i}}\right)-\eta^{\prime}\left(\text { Chap }^{\mathrm{i}}\right)\right] \cdot \operatorname{aerosol}^{\mathrm{i}} \\
& \frac{\partial}{\partial \eta^{\prime}} \chi^{2}=-2 \cdot \sum_{\mathrm{i}}^{\mathrm{K}}\left[\Delta \mathrm{a}_{\text {total }}^{\mathrm{i}}-\rho\left(\text { Rayleigh }^{\mathrm{i}}\right)-\alpha\left(\text { aerosol }^{\mathrm{i}}\right)-\eta^{\prime}\left(\text { Chap }^{\mathrm{i}}\right)\right] \cdot \text { Chap }^{\mathrm{i}}
\end{aligned}
$$

Set the partial derivatives equal to zero and rewrite as :

$$
\begin{aligned}
& \sum_{i}^{K} \Delta a_{\text {total }}^{i} \cdot \text { Rayleigh }^{\mathrm{i}}=\rho \sum_{i}^{\mathrm{K}}\left(\text { Rayleigh }^{\mathrm{i}}\right)^{2}+\alpha \sum_{i}^{\mathrm{K}}\left(\text { aerosol }^{\mathrm{i}} \cdot \text { Rayleigh }^{\mathrm{i}}\right)+\eta^{\prime} \sum_{\mathrm{i}}^{\mathrm{K}}\left(\mathrm{Chap}^{\mathrm{i}} \cdot \text { Rayleigh }^{\mathrm{i}}\right) \\
& \sum_{i}^{K} \Delta a_{\text {total }}^{i} \cdot \operatorname{aerosol}^{i}=\rho \sum_{i}^{K}\left(\text { Rayleigh }^{i} \cdot \operatorname{aerosol}^{i}\right)+\alpha \sum_{i}^{K}\left(\operatorname{aerosol}^{i^{i}}\right)^{2}+\eta^{\prime} \sum_{i}^{K}\left(\text { Chap }^{i} \cdot \operatorname{aerosol}^{i}\right) \\
& \sum_{i}^{K} \Delta a_{\text {total }}^{i} \cdot \text { Chap }^{i}=\rho \sum_{i}^{K}\left(\text { Rayleigh }^{i} \cdot \text { Chap }^{i}\right)+\alpha \sum_{i}^{K}\left(\text { aerosol }^{i} \cdot \text { Chap }^{i}\right)+\eta^{\prime} \sum_{i}^{K}\left(\text { Chap }^{i}\right)^{2} \text {. }
\end{aligned}
$$

Each summation in the equations can be calculated. The summations in Equation [57] are :

$$
\begin{gathered}
\sum_{\mathrm{i}}^{\mathrm{K}} \Delta \mathrm{a}_{\text {total }}^{\mathrm{i}} \cdot \text { Rayleigh }^{\mathrm{i}}=\Psi_{1} \\
\sum_{\mathrm{i}}^{\mathrm{K}}\left(\text { Rayleigh }^{\mathrm{i}}\right)^{2}=\mathrm{x}_{1}
\end{gathered}
$$




$$
\begin{aligned}
& \sum_{i}^{K}\left(\operatorname{aerosol}^{i} \cdot \text { Rayleigh }^{\mathbf{i}}\right)=y_{1} \\
& \sum_{i}^{K}\left(\text { Chap }^{i} \cdot \text { Rayleigh }^{i}\right)=z_{1}
\end{aligned}
$$

The summations in Equation [58] are :

$$
\begin{gathered}
\sum_{i}^{K} \Delta a_{\text {total }}^{i} \cdot \operatorname{aerosol}^{i}=\Psi_{2} \\
\sum_{i}^{K}\left(\text { Rayleigh }^{\mathrm{i}} \cdot \operatorname{aerosol}^{\mathrm{i}}\right)=\mathrm{x}_{2} \\
\sum_{\mathrm{i}}^{\mathrm{K}}\left(\operatorname{aerosol}^{\mathrm{i}^{\mathrm{i}}}\right)^{2}=\mathrm{y}_{2} \\
\sum_{\mathrm{i}}^{\mathrm{K}}\left(\text { Chap }^{\mathrm{i}} \cdot \operatorname{aerosol}^{\mathrm{i}}\right)=\mathrm{z}_{2} .
\end{gathered}
$$

The summations in Equation [59] are :

$$
\begin{gathered}
\sum_{i}^{K} \Delta a_{\text {total }}^{i} \cdot \text { Chap }^{i}=\Psi_{3} \\
\sum_{i}^{K}\left(\operatorname{Rayleigh}^{i} \cdot \text { Chap }^{i}\right)=x_{3} \\
\sum_{i}^{K}\left(\operatorname{aerosol}^{i} \cdot \text { Chap }^{i}\right)=y_{3} \\
\sum_{i}^{K}\left(\text { Chap }^{i^{2}}\right)^{2}=z_{3} .
\end{gathered}
$$

Replacing the summation designations given above back into Equations [57-59] gives three equations in three unknowns $\left(\rho, \alpha, \eta^{\prime}\right)$ : 


$$
\begin{aligned}
& \Psi_{1}=\rho x_{1}+\alpha y_{1}+\eta^{\prime} z_{1} \\
& \Psi_{2}=\rho x_{2}+\alpha y_{2}+\eta^{\prime} z_{2} \\
& \Psi_{3}=\rho x_{3}+\alpha y_{3}+\eta^{\prime} z_{3} .
\end{aligned}
$$

Rewrite the above set of equations in matrix notation (note the matrix is symmetric):

$$
\begin{gathered}
{\left[\begin{array}{l}
\Psi_{1} \\
\Psi_{2} \\
\Psi_{3}
\end{array}\right]=\left[\begin{array}{lll}
\mathrm{x}_{1} & \mathrm{y}_{1} & \mathrm{z}_{1} \\
\mathrm{x}_{2} & \mathrm{y}_{2} & \mathrm{z}_{2} \\
\mathrm{x}_{3} & \mathrm{y}_{3} & \mathrm{z}_{3}
\end{array}\right]\left[\begin{array}{c}
\rho \\
\alpha \\
\eta^{\prime}
\end{array}\right]} \\
\mathrm{x}_{2}=\mathrm{y}_{1} ; \mathrm{x}_{3}=\mathrm{z}_{1} ; \mathrm{y}_{3}=\mathrm{z}_{2} .
\end{gathered}
$$

Solve for $\rho, \alpha$, and $\eta^{\prime}$ by Gaussian elimination.

The path corrected ozone column depth $\eta$ is :

$$
\eta=\eta^{\prime} \cdot \cos \theta \cdot \cos \phi
$$

The above correction takes into consideration the longer optical path through the atmosphere for non-zenith observations. The variable $\theta$ is the star hour angle from zenith at exposure and $\phi$ is the angular difference between the star declination and the observatory latitude. 


\section{ANALYSIS}

The validity of values $\rho, \alpha$, and $\eta$ are based on knowledge of the behavior of Rayleigh scattering, aerosol scattering, and ozone absorption with wavelength. Rayleigh scattering attenuates radiation more strongly in the shorter wavelengths. The Rayleigh scattering extinction coefficient, $\rho$, should be a positive value since Rayleigh scattering extinction varies as the inverse fourth power of wavelength. Aerosol scattering also favors attenuation of shorter wavelengths. The aerosol scattering extinction coefficient, $\alpha$, should be a negative value because aerosol scattering extinction is a linear function of wavelength. The sum of Rayleigh and aerosol scattering extinctions must, overall, attenuate radiation more strongly towards the shorter wavelengths, although individually, $\rho$ or $\alpha$ may suggest stronger extinction towards longer wavelengths due to the limited wavelength range of the data suitable for curve fitting. Ozone abundance, $\eta$, must be positive or zero for any meaningful interpretation. If these conditions are not met the validity of the measurement is suspect.

Uncertainties in physical measurements determine the weight of validity accorded to the results of the ozone measurement method. Uncertainties of the photographic emulsion sensitivity curve, stellar radiation energies, and Chappuis absorption coefficients are factors that affect the column depth uncertainty.

The errors involved in this ozone determination are the result of measurement uncertainties. The theory of error propagation is used to calculate the error incurred by this method. Begin with the measurement of stellar intensity. The uncertainty of the intensity, $\delta \log \mathrm{J}_{\text {below }}$, is derived from Equation [32] : 


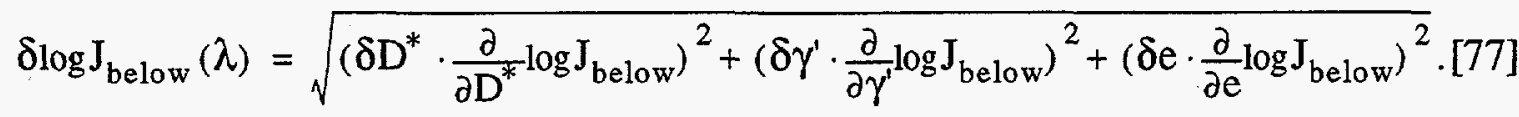

$\delta D^{*}$ is the measurement uncertainty of the stellar strip density. $\delta \gamma^{\prime}$ is the uncertainty of the characteristic curve contrast. $\delta \mathrm{e}$ is the plate sensitivity uncertainty. The partial derivatives from the above uncertainty are :

$$
\begin{gathered}
\frac{\partial}{\partial D^{*}} \log \mathrm{J}_{\text {below }}=\frac{1}{\gamma^{\prime}(\lambda)} \\
\frac{\partial}{\partial \gamma^{\prime}} \log \mathrm{J}_{\text {below }}=\frac{0.6-D^{*}(\lambda)}{\gamma^{\prime}(\lambda)^{2}} \\
\frac{\partial}{\partial e} \log \mathrm{J}_{\text {below }}=-1 .
\end{gathered}
$$

The intensity uncertainty becomes :

$$
\delta \log \mathrm{J}_{\text {below }}(\lambda)=\sqrt{\left(\delta \mathrm{D}^{*} \cdot \frac{1}{\gamma^{\prime}(\lambda)}\right)^{2}+\left(\delta \gamma^{\prime} \cdot \frac{0.6-\mathrm{D}^{*}(\lambda)}{\gamma^{\prime}(\lambda)^{2}}\right)^{2}+(\delta \mathrm{e})^{2}} .
$$

The dominant term in the intensity uncertainty is the sensitivity uncertainty, $\delta$ e. The intensity uncertainty reduces to :

$$
\delta \log \mathrm{J}_{\text {below }} \approx \delta \mathrm{e}=0.01
$$

Sensitivity $e(\lambda)$ varies with each plate. Direct photometric measurements of plate sensitivity is determined from the plate's response to a calibrated lamp. However, the plate sensitivity used in this measurement is from Kodak (1994) since the energy distribution of the calibration lamp is unknown. The values from Kodak are average plate sensitivities. The sensitivity uncertainty $\delta e$ is the uncertainty of the Kodak data. 
The next level of error propagation is the error associated with the extinction $a_{\text {total }}$. Recall Equation [37] and write the extinction uncertainty $\delta a$ as :

$$
\delta a_{\text {total }}=\sqrt{\left(\delta \log \Phi_{E T} \cdot \frac{\partial a_{\text {total }}}{\partial \log \Phi_{E T}}\right)^{2}+\left(\delta \log J_{\text {below }} \cdot \frac{\partial a_{\text {total }}}{\partial \log J_{\text {below }}}\right)^{2}}
$$

The partial derivatives from the above uncertainty are :

$$
\begin{gathered}
\frac{\partial a_{\text {total }}}{\partial\left(\log \Phi_{E T}\right)}=1 \\
\frac{\partial a_{\text {total }}}{\partial\left(\log J_{\text {below }}\right)}=-1
\end{gathered}
$$

The extinction uncertainty becomes :

$$
\delta a_{\text {total }}=\sqrt{\left(\delta \log \Phi_{E T}\right)^{2}+\left(\delta \log J_{\text {below }}\right)^{2}}
$$

The uncertainty of $\log \Phi_{\mathrm{ET}}, \delta \log \Phi_{\mathrm{ET}}$, is determined by the uncertainty of Glushneva's extra-terrestrial data $\delta \Phi_{\mathrm{ET}}$ :

$$
\delta \log \Phi_{\mathrm{ET}}=\log e \cdot \frac{\delta \Phi_{\mathrm{ET}}}{\Phi_{\mathrm{ET}}} \approx 0.0001
$$

The extinction uncertainty $\delta$ a simplifies to :

$$
\delta a_{\text {total }}=\sqrt{\left(\delta \log \Phi_{E T}\right)^{2}+(\delta e)^{2}} \approx \delta \mathrm{e} .
$$


The uncertainty in the differential total extinction $\Delta \mathrm{a}_{\text {total }}$ is calculated by applying error propagation to Equation [48] :

$$
\delta\left(\Delta \mathrm{a}_{\text {total }}\right)=\sqrt{\left(\delta \mathrm{a}_{\text {total }}\left(\lambda_{\mathrm{n}}\right) \cdot \frac{\partial}{\partial \mathrm{a}_{\text {total }}\left(\lambda_{\mathrm{n}}\right)} \Delta \mathrm{a}_{\text {total }}\right)^{2}+\left(\delta \mathrm{a}_{\text {total }}\left(\lambda_{\mathrm{m}}\right) \cdot \frac{\partial}{\partial \mathrm{a}_{\text {total }}\left(\lambda_{\mathrm{m}}\right)} \Delta \mathrm{a}_{\text {total }}\right)^{2}}
$$

The partial derivatives from the above uncertainty are :

$$
\begin{gathered}
\frac{\partial}{\partial a_{\text {total }}\left(\lambda_{n}\right)} \Delta a_{\text {total }}=1 \\
\frac{\partial}{\partial a_{\text {total }}\left(\lambda_{\mathrm{m}}\right)} \Delta \mathrm{a}_{\text {total }}=-1
\end{gathered}
$$

The differential total extinction uncertainty becomes :

$$
\delta\left(\Delta \mathrm{a}_{\text {total }}\right)=\sqrt{\left(\delta \mathrm{a}_{\text {total }}\left(\lambda_{\mathrm{n}}\right)\right)^{2}+\left(\delta \mathrm{a}_{\text {total }}\left(\lambda_{\mathrm{m}}\right)\right)^{2}}=\sqrt{2} \cdot \delta \mathrm{a}_{\text {total }}
$$

Replace $\delta a_{\text {total }}$ for $\delta e:$

$$
\delta\left(\Delta \mathrm{a}_{\text {total }}\right)=\sqrt{2} \cdot \delta \mathrm{a}_{\text {total }}=\sqrt{2} \cdot \delta \mathrm{e}
$$

The calculation of ozone column depth uncertainty begins by substituting the Rayleigh and aerosol scattering coefficients $\rho$ and $\alpha$ back into Equation [59] and solve for column depth $\eta^{\prime}$ :

$$
\eta^{\prime}=\frac{\sum_{i}^{K}\left[\Delta a_{\text {total }}^{i}-\rho\left(\text { Rayleigh }^{i}\right)-\alpha\left(\text { aerosol }^{i}\right)\right] \cdot\left(\text { Chap }^{i}\right)}{\sum_{i}^{K}\left(\text { Chap }^{i}\right)^{2}} .
$$


Define a new differential total extinction $\Delta \mathrm{a}^{\mathrm{i}}$ without Rayleigh and aerosol scattering :

$$
\Delta \mathrm{a}^{\mathrm{i}} \equiv \Delta \mathrm{a}_{\text {total }}^{\mathrm{i}}-\rho\left(\text { Rayleigh }^{\mathrm{i}}\right)-\alpha\left(\text { aerosol }^{\mathrm{i}}\right)=\eta^{\prime} \cdot\left(\text { Chap }^{\mathrm{i}}\right) .
$$

Rewrite the ozone column depth as :

$$
\eta^{\prime}=\frac{\sum_{i}^{K} \Delta a^{i} \cdot\left(\text { Chap }^{i}\right)}{\sum_{i}^{K}\left(\text { Chap }^{i^{i}}\right)^{2}} .
$$

The uncertainty of the ozone column depth $\eta^{\prime}$ is :

$$
\begin{aligned}
\delta \eta^{\prime} & =\sqrt{\sum_{i}^{K}\left(\delta\left(\Delta a^{i}\right) \cdot \frac{\partial \eta^{\prime}}{\partial\left(\Delta a^{i}\right)}\right)^{2}+\sum_{i}^{K}\left(\delta(\text { Chap }) \cdot \frac{\partial \eta^{\prime}}{\partial\left(\text { Chap }^{i}\right)}\right)^{2}} \\
& =\sqrt{\left(\delta\left(\Delta a_{\text {total }}\right)\right)^{2} \cdot \sum_{i}^{K}\left(\frac{\partial \eta^{\prime}}{\partial\left(\Delta a^{i}\right)}\right)^{2}+(\delta(\text { Chap }))^{2} \cdot \sum_{i}^{K}\left(\frac{\partial \eta^{\prime}}{\partial(\text { Chap })}\right)^{i}} .
\end{aligned}
$$

$\delta($ Chap) is the uncertainty of the Chappuis band coefficient differential :

$$
\delta(\text { Chap })=\sqrt{\left(\delta \kappa\left(\lambda_{\mathrm{n}}\right)\right)^{2}+\left(\delta \kappa\left(\lambda_{\mathrm{m}}\right)\right)^{2}}=\sqrt{2} \cdot \delta \kappa \approx 0.001 .
$$

$\delta \kappa$ is the uncertainty of the Chappuis band coefficients (Inn and Tanaka, 1953).

The partial derivatives from the column depth uncertainty are : 


$$
\begin{gathered}
\frac{\partial \eta^{\prime}}{\partial\left(\Delta a^{i}\right)}=\frac{\left(\text { Chap }^{i}\right)}{\sum_{j}\left(\text { Chap }^{j}\right)^{2}} \\
\frac{\partial \eta^{\prime}}{\partial\left(\text { Chap }^{i}\right)}=\frac{\Delta a^{i}}{\sum_{j}^{K}\left(\text { Chap }^{j}\right)^{2}}-\frac{2 \cdot \eta^{\prime} \cdot\left(\text { Chap }^{i}\right)}{\sum_{j}^{K}\left(\text { Chap }^{j^{j}}\right)^{2}}
\end{gathered}
$$

Recall Equation [95] and replace $\Delta \mathrm{a}^{\mathrm{i}}$ with $\eta^{\prime}\left(\mathrm{Chap}^{\mathrm{i}}\right)$ in the above equation :

$$
\begin{aligned}
\frac{\partial \eta^{\prime}}{\partial\left(\text { Chap }^{i}\right)} & =\frac{\Delta a^{i}}{\sum_{j}^{K}\left(\text { Chap }^{j^{2}}\right)^{2}}-\frac{2 \cdot \eta^{\prime} \cdot\left(\text { Chap }^{i}\right)}{\sum_{j}^{K}\left(\text { Chap }^{j^{2}}\right)^{2}} \\
& =\frac{\eta^{\prime} \cdot\left(\text { Chap }^{i}\right)}{\sum_{j}^{K}\left(\text { Chap }^{j^{2}}\right)^{2}}-2 \cdot \frac{\eta^{\prime} \cdot\left(\text { Chap }^{i}\right)}{\sum_{j}^{K}\left(\text { Chap }^{j^{2}}\right)^{2}} \\
& =-\frac{\eta^{\prime} \cdot\left(\text { Chap }^{i}\right)}{\sum_{j}^{K}\left(\text { Chap }^{j^{2}}\right)^{2}}
\end{aligned}
$$

The ozone column depth uncertainty reduces to :

$$
\begin{aligned}
& \delta \eta^{\prime}=\sqrt{\left(\delta\left(\Delta \mathrm{a}_{\text {total }}\right)\right)^{2} \cdot \sum_{\mathrm{i}}^{\mathrm{K}}\left(\frac{\left(\mathrm{Chap}^{\mathrm{i}}\right)}{\left.\sum_{\mathrm{j}}^{\mathrm{K}\left(\mathrm{Chap}^{\mathrm{j}}\right)^{2}}\right)^{2}}\right)^{2}+(\delta(\text { Chap }))^{2} \cdot \sum_{\mathrm{i}}^{\mathrm{K}}\left(\frac{\eta^{\prime} \cdot\left(\mathrm{Chap}^{\mathrm{i}}\right)}{\left.\sum_{\mathrm{j}}^{\mathrm{K}\left(\mathrm{Chap}^{\mathrm{j}}\right)^{2}}\right)^{2}}\right.} \\
& =\sqrt{\left[\left(\delta\left(\Delta a_{\text {total }}\right)\right)^{2}+\left(\eta^{\prime} \cdot \delta(\text { Chap })\right)^{2}\right] \cdot \frac{\sum_{i}^{K}\left(\text { Chap }^{i}\right)^{2}}{\left(\sum_{j}^{K}\left(\text { Chap }^{j}\right)^{2}\right)^{2}}}
\end{aligned}
$$




$$
\delta \eta^{\prime}=\sqrt{\frac{\left(\delta\left(\Delta \mathrm{a}_{\text {total }}\right)\right)^{2}+\left(\eta^{\prime} \cdot \delta(\text { Chap })\right)^{2}}{\sum_{i}^{K}\left(\operatorname{Chap}^{i}\right)^{2}}} .
$$

Replace $\delta\left(\Delta \mathrm{a}_{\text {total }}\right)$ with Equation [93] and $\delta($ Chap) with Equation [98] for the above equation. The column depth uncertainty further simplifies to :

$$
\delta \eta^{\prime}=\sqrt{2 \cdot \frac{(\delta e)^{2}+\left(\eta^{\prime} \cdot \delta \kappa\right)^{2}}{\sum_{i}^{K}\left(\operatorname{Chap}^{i}\right)^{2}}} .
$$

The sensitivity uncertainty is the dominant term :

$$
\delta \eta^{\prime}=\delta e \cdot \sqrt{\frac{2}{\sum_{i}^{K}\left(\text { Chap }^{i}\right)^{2}}} .
$$

The Chappuis coefficient differential Chap ${ }^{i}$ minimizes the column depth uncertainty $\delta \eta^{\prime}$ when Chap ${ }^{i}$ is at the maximum possible values. The experimental interpretation of this rule is to choose Chappuis coefficients $\kappa\left(\lambda_{n}\right)$ and $\kappa\left(\lambda_{m}\right)$ such that their difference is maximized within the given wavelength range of the data. Therefore only differentials Rayleigh $^{\mathrm{i}}$, aerosol ${ }^{\mathrm{i}}$, and $\Delta \mathrm{a}_{\text {total }}^{\mathrm{i}}$ along with the corresponding Chappuis coefficient differential meeting the minimal value are used.

In light of this uncertainty analysis, the number of possible data point pairs $\mathrm{K}$ is redefined as the number of pairs of data points used for minimum column depth uncertainty. The other factors of the column depth uncertainty are invariant of the methodology. Of these 
factors, the plate sensitivity uncertainty $\delta \mathrm{e}$ is the dominant term contributing to the column depth uncertainty.

The minimum column depth uncertainty is derived from the ozone extinction formula :

$$
J(\lambda)=J_{0}(\lambda) \cdot 10^{-\kappa(\lambda) \cdot \eta^{\prime}}
$$

Take the logarithm of the equation and find the differential for $\lambda_{n}$ and $\lambda_{m}$ :

$$
\log J\left(\lambda_{n}\right)-\log J\left(\lambda_{m}\right)=\log J_{0}\left(\lambda_{n}\right)-\log J_{0}\left(\lambda_{m}\right)-\eta \cdot\left(\kappa\left(\lambda_{n}\right)-\kappa\left(\lambda_{m}\right)\right)
$$

The value $\log J\left(\lambda_{n}\right)-\log J\left(\lambda_{m}\right)$ must be equal to or greater than the intensity uncertainty, $\delta \log \mathrm{J}_{\text {below }}$, for a valid measurement. Rewrite the above equation as :

$$
\begin{aligned}
\delta \log \mathrm{J}_{\text {below }} & \leq\left|\log \mathrm{J}\left(\lambda_{\mathrm{n}}\right)-\log \mathrm{J}\left(\lambda_{\mathrm{m}}\right)\right| \\
& \leq\left|\log \mathrm{J}_{\mathrm{o}}\left(\lambda_{\mathrm{n}}\right)-\log \mathrm{J}_{\mathrm{o}}\left(\lambda_{\mathrm{m}}\right)-\eta \cdot\left(\kappa\left(\lambda_{\mathrm{n}}\right)-\kappa\left(\lambda_{\mathrm{m}}\right)\right)\right| .
\end{aligned}
$$

Since the measurement is relevant to the differential amount of Chappuis attenuation, assume $J_{0}\left(\lambda_{n}\right)$ and $J_{0}\left(\lambda_{m}\right)$ are equivalent to derive the Chappuis differential extremum :

$$
\delta \log J_{\text {below }} \leq\left|\eta \cdot\left(\kappa\left(\lambda_{n}\right)-\kappa\left(\lambda_{m}\right)\right)\right|
$$

Recall $\kappa\left(\lambda_{n}\right)-\kappa\left(\lambda_{m}\right)=$ Chap $^{i}$. Find the minimal limit of the Chappuis coefficient differential :

$$
\mid \text { Chap }^{\mathrm{i}} \mid \geq \frac{\delta \log \mathrm{J}_{\text {below }}}{\bar{\eta}}
$$


The lower limit of Chap ${ }^{i}$ is determined by the intensity uncertainty, $\delta \log \mathrm{J}_{\text {below }}$, and the average ozone column depth, $\bar{\eta}$. The lower limit of $\mathrm{Chap}^{\mathrm{i}}$ is $0.03 \mathrm{~atm}-\mathrm{cm}^{-1}$. Unfortunately, this lower limit exceeds the range available in the data. Let the lower limit for Chap ${ }^{i}$ be $0.01 \mathrm{~atm}-\mathrm{cm}$. This lower limit restricts the number of possible pair combinations $\mathrm{K}$ therefore setting a minimum possible value for the column depth uncertainty.

The uncertainty $\delta \eta$ due to the hour angle uncertainty, $\delta \theta$, and declination uncertainty, $\delta \phi$, is:

$$
\begin{aligned}
\delta \eta & =\sqrt{\left(\delta \eta^{\prime} \cdot \frac{\partial \eta}{\partial \eta^{\prime}}\right)^{2}+\left(\delta \theta \cdot \frac{\partial \eta}{\partial \theta}\right)^{2}+\left(\delta \phi \cdot \frac{\partial \eta}{\partial \phi}\right)^{2}} \\
& =\sqrt{\left(\delta \eta^{\prime} \cdot \cos \theta \cdot \cos \phi\right)^{2}+\left(\delta \theta \cdot \eta^{\prime} \cdot \sin \theta \cdot \cos \phi\right)^{2}+\left(\delta \phi \cdot \eta^{\prime} \cdot \cos \theta \cdot \sin \phi\right)^{2}}
\end{aligned}
$$

The hour angle $\theta$ is $30^{\circ}$ with an uncertainty $\delta \theta$ of $1^{\circ}$. The declination difference, $\phi$, is $6^{\circ}$ with an uncertainty $\delta \phi$ of $1^{\circ}$.

The possible range of values for vertical ozone column depth is 0.200 to $0.600 \mathrm{~atm}-\mathrm{cm}$ (Lacis and Hansen, 1974). At Lick Observatory's latitude $\left(\sim 37^{\circ} \mathrm{N}\right)$, the recorded long term (1958-1980) monthly averages span from a high of $0.370 \mathrm{~atm}-\mathrm{cm}$ in March to a low of $0.295 \mathrm{~atm}-\mathrm{cm}$ in September (Schachter, 1991). By the method and analysis presented in this thesis, the vertical ozone column depth for Lick Observatory on September 11, 1963 as measured from an astronomical spectrographic plate was $\eta=0.263$ atm-cm with an uncertainty of $\delta \eta=0.173 \mathrm{~atm}-\mathrm{cm}$. The mean monthly column depth is approximately $0.290 \mathrm{~atm}-\mathrm{cm}$ (Cicerone, 1987). According to the data from Rindert (1976), observed daily fluctuations of $20 \%$ about the monthly average from Uppsala, Sweden $\left(69^{\circ} \mathrm{N}\right)$ indicate the possibility of large variations for day to day measurements (Schachter, 1991). 


\section{CONCLUSION}

When judging the usefulness of this ozone measurement method, spatial and temporal considerations must be made. Daily ozone column depths can fluctuate greatly about the monthly average (Rindert, 1976). This measurement of ozone column depth is representative only of the observatory site and not of a greater geographic area. The plate is a record of ozone column depth for a particular place and time.

This method of measuring ozone column depth from stellar spectrographic plates should be applied in the context of tracking past ozone abundances. The errors associated with this measurement are too great for precise determination of ozone abundance. This method is intended for use in monitoring global ozone abundance trends. An uncertainty of $10 \%(0.030 \mathrm{~atm}-\mathrm{cm})$ is acceptable for climatic studies involving ozone (Grossman, 1995).

This study is intended as a preliminary study of a novel approach to ozone column depth measurement. The goal of the study is to determine the feasibility of extracting an ozone column depth with an acceptable error from stellar spectrographic plates taken at earlier times. Given the aforementioned conditions under which this study was performed, refinements need to be made.

An improvement to the study is verification of ozone column depth with independent measurements conducted at the same time and place. Other than comparing the results of this method to spatial and temporal averages of ozone column depth, there is no sufficiently detailed source to verify a single measurement. In future development of this 
method, the spectrographic plate used to test the method should be chosen when there is simultaneous ozone data recorded for that date and location.

A lower resolution, wider band spectrographic plate would serve this method better. Atmospheric absorption bands are typically much wider than stellar and interstellar absorption bands. A low resolution, wide band spectrographic plate would have reduced much of the high frequency noise and included more of the Chappuis ozone absorption band. It is possible to decrease in the column depth uncertainty by using the wider range of Chappuis coefficients available to maximize the differential Chap ${ }^{i}$.

The energy distribution of the calibration lamp must be determined to improve the modeling of the photographic characteristic curve and to provide a meaningful measurement. In astronomical spectroscopy, the absolute energy distribution of the lamp is not relevant to the measurement and usually is not recorded. No records of the energy distribution or the manufacturer of the lamp used on this plate were found at Lick Observatory. Data of the lamp energy distribution are used to confirm whether conversion from density to energy is successful. The uncertainty of the lamp energy distribution (i.e. plate sensitivity uncertainty) is the greatest uncertainty in this method of ozone column depth measurement.

Better models of aerosol and Rayleigh scattering could improve the measurement. The presently used scattering models are too simplistic for finer detail measurements. A comparison of this method's results with the results from a model atmosphere attenuation would also help refine this measurement (Grossman et al., 1993; Grossman and Grant, 1994ab). A model atmosphere can be used to provide a more detailed analysis of the 
attenuation by the Chappuis band as the radiation penetrates the atmosphere.

An improvement of the error could be achieved by reducing the uncertainty of the sensitivity. At present, the sensitivity uncertainty is the dominant term in the error analysis. A reduction of the sensitivity uncertainty by a factor of 10 would reduce the column depth uncertainty also by a factor of 10 , thereby achieving the desired maximum $10 \%$ column depth uncertainty for climatology use (Grossman, 1995). The manufacturers of spectrographic plates may have more accurate sensitivity curves than the plate's technical specification sheet used in this thesis.

Should this method be developed into an acceptable ozone column depth measurement program, the implementation should take into consideration the capabilities and limitations. This method does not rival other measurements in accuracy and should not be viewed as a method for present day ozone measurements. The value is in measurement of historical ozone column depths by virtue of the fact that old stellar spectrographic plates are records of past atmospheric transmission conditions. This method can be used to extend ozone data from pre-Dobson instrument era (1920s) to the dawn of photographic stellar spectroscopy (1900s). 
Table 1: Relative intensities of strips.

\begin{tabular}{|c|c|}
\hline $\begin{array}{c}\text { strip } \\
\text { number } n\end{array}$ & $\log (\mathrm{n})$ \\
\hline \hline 1 & 0.752 \\
\hline 2 & 1.027 \\
\hline 3 & 1.350 \\
\hline 4 & 1.656 \\
\hline 5 & 1.955 \\
\hline 6 & 2.255 \\
\hline
\end{tabular}




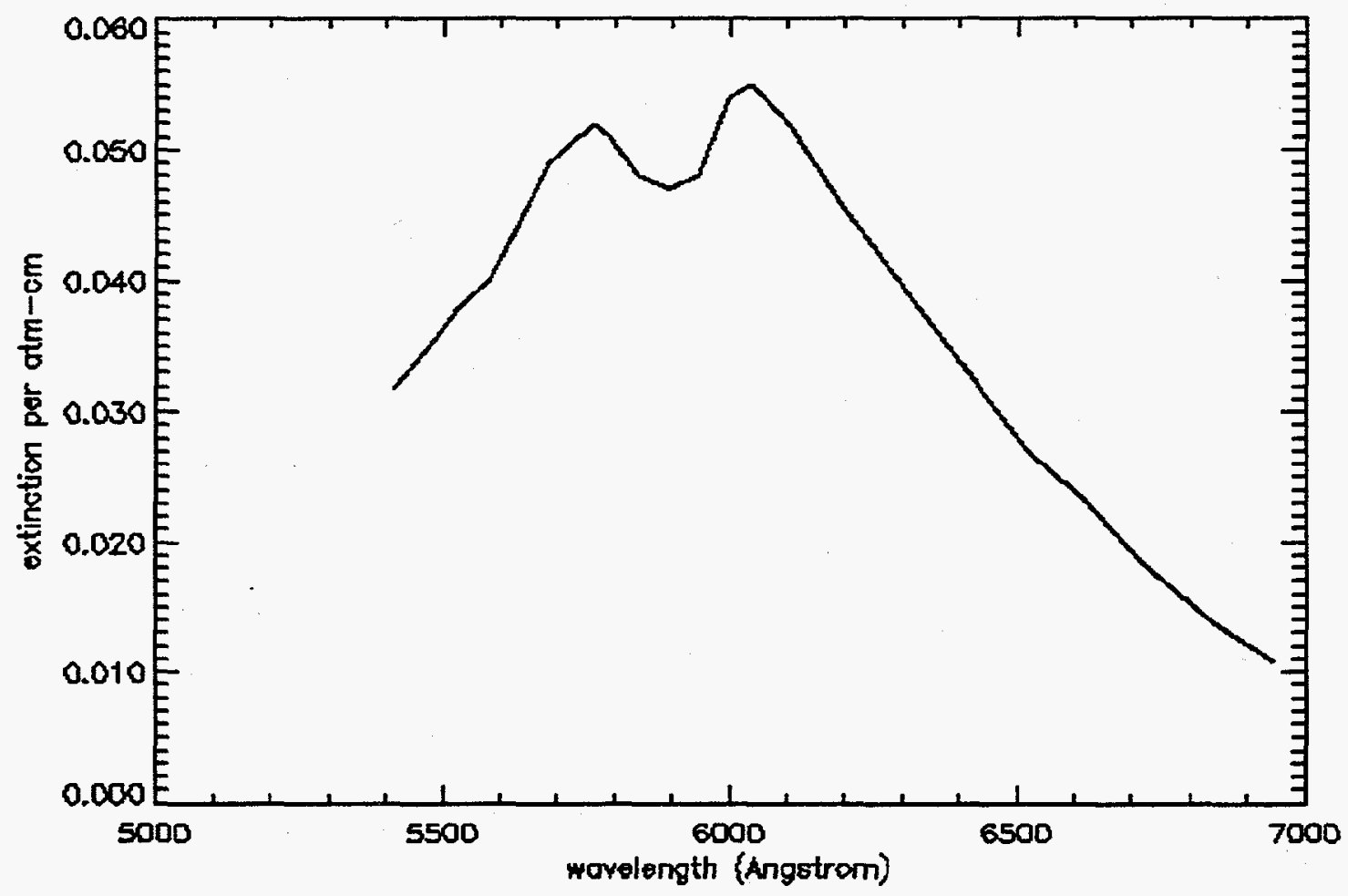

Figure 1 : Chappuis band absorption coefficients. 


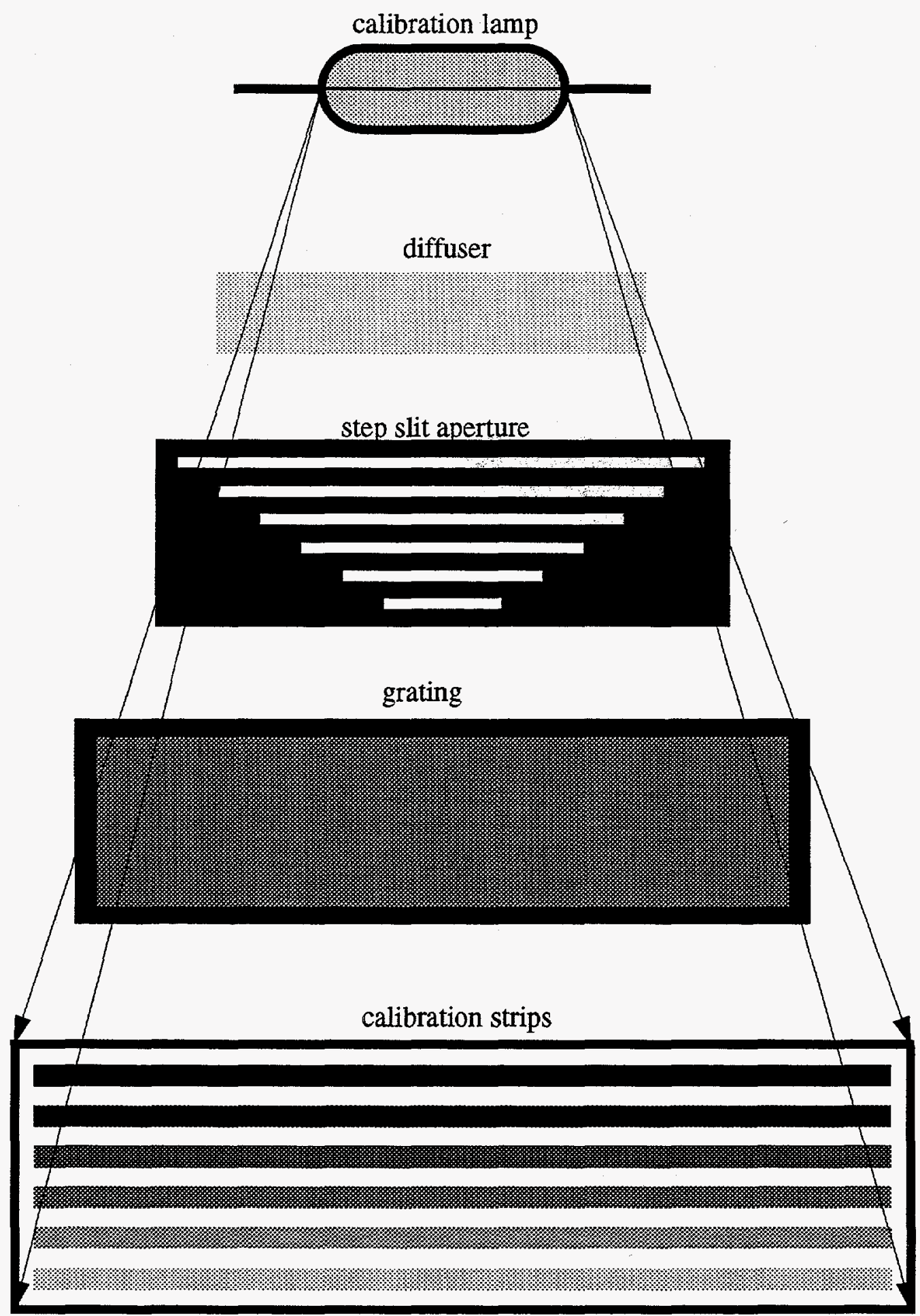

Figure 2 : Calibration of plate by step slit aperature. 


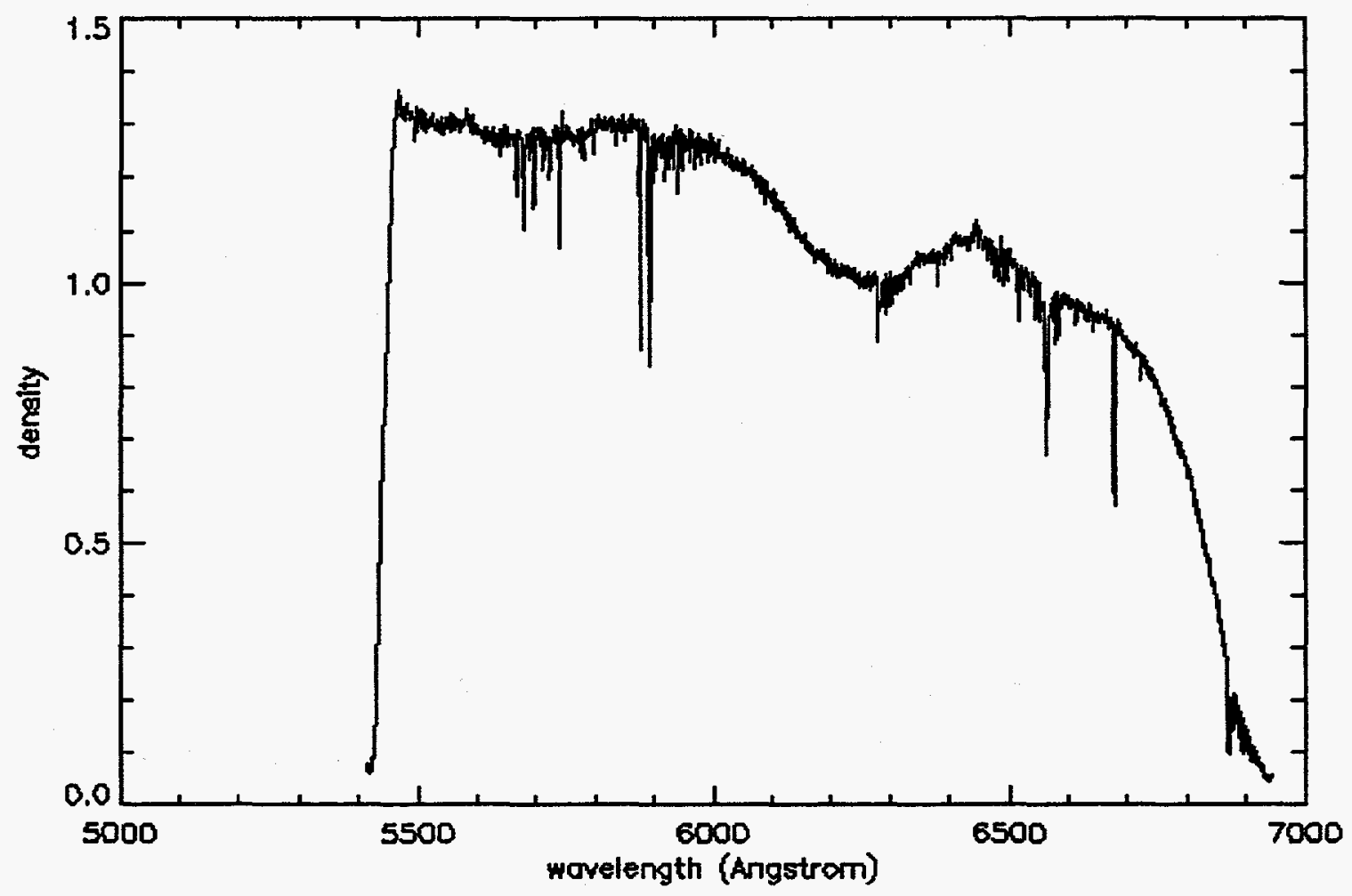

Figure 3 : Stellar strip density. 


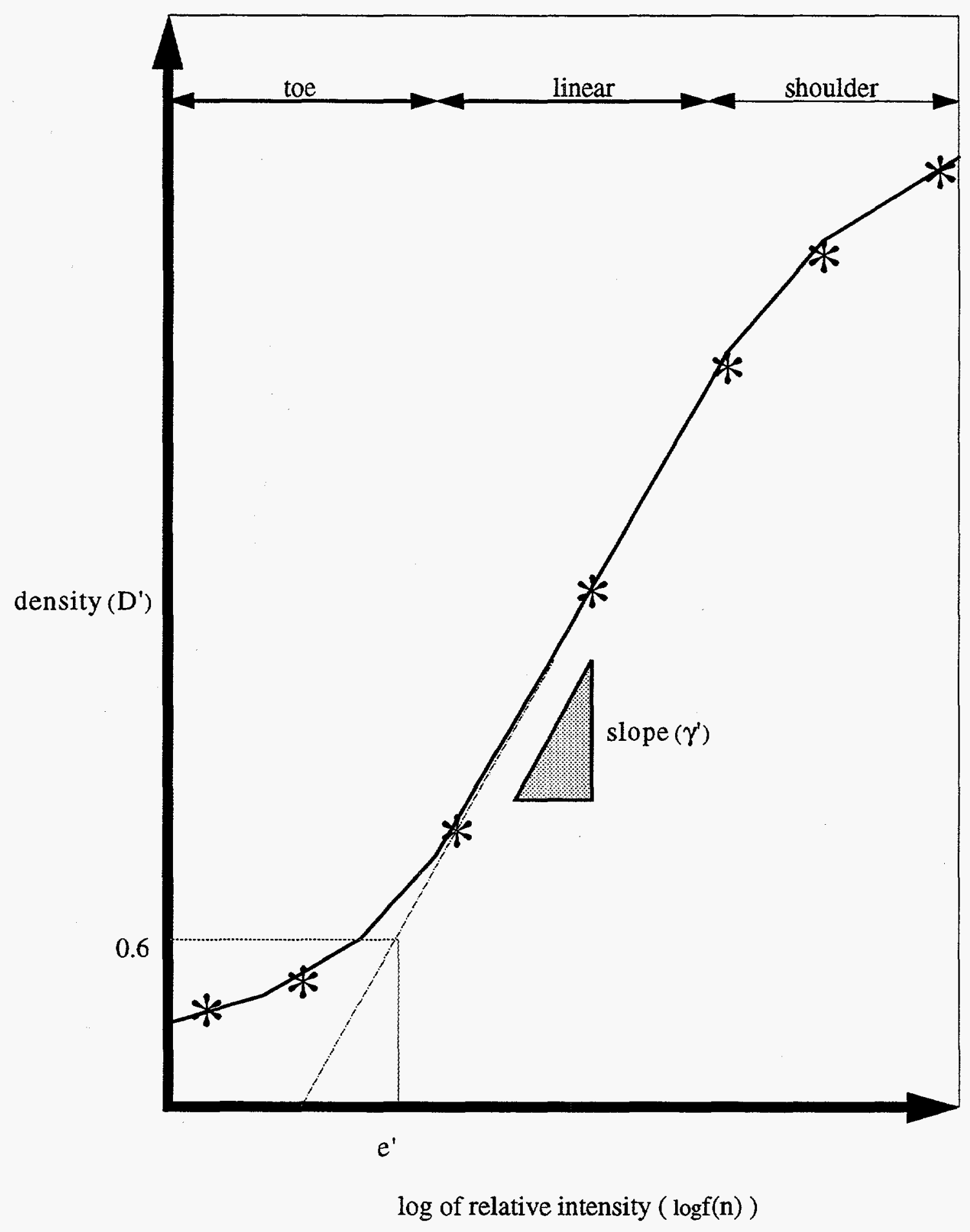

Figure 4 : characteristic curve for a wavelength $\lambda$. 


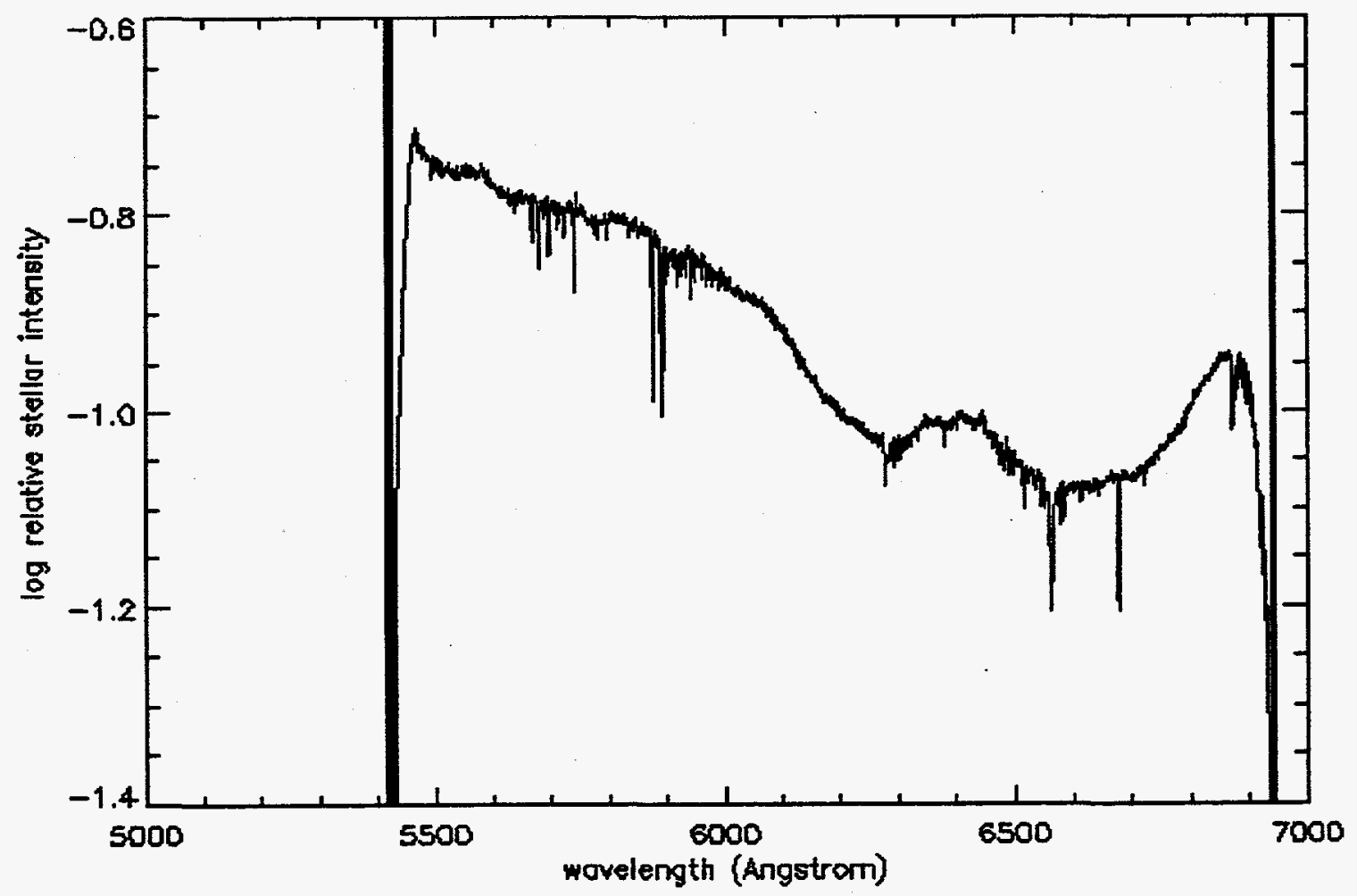

Figure 5 : Relative stellar intensity. 


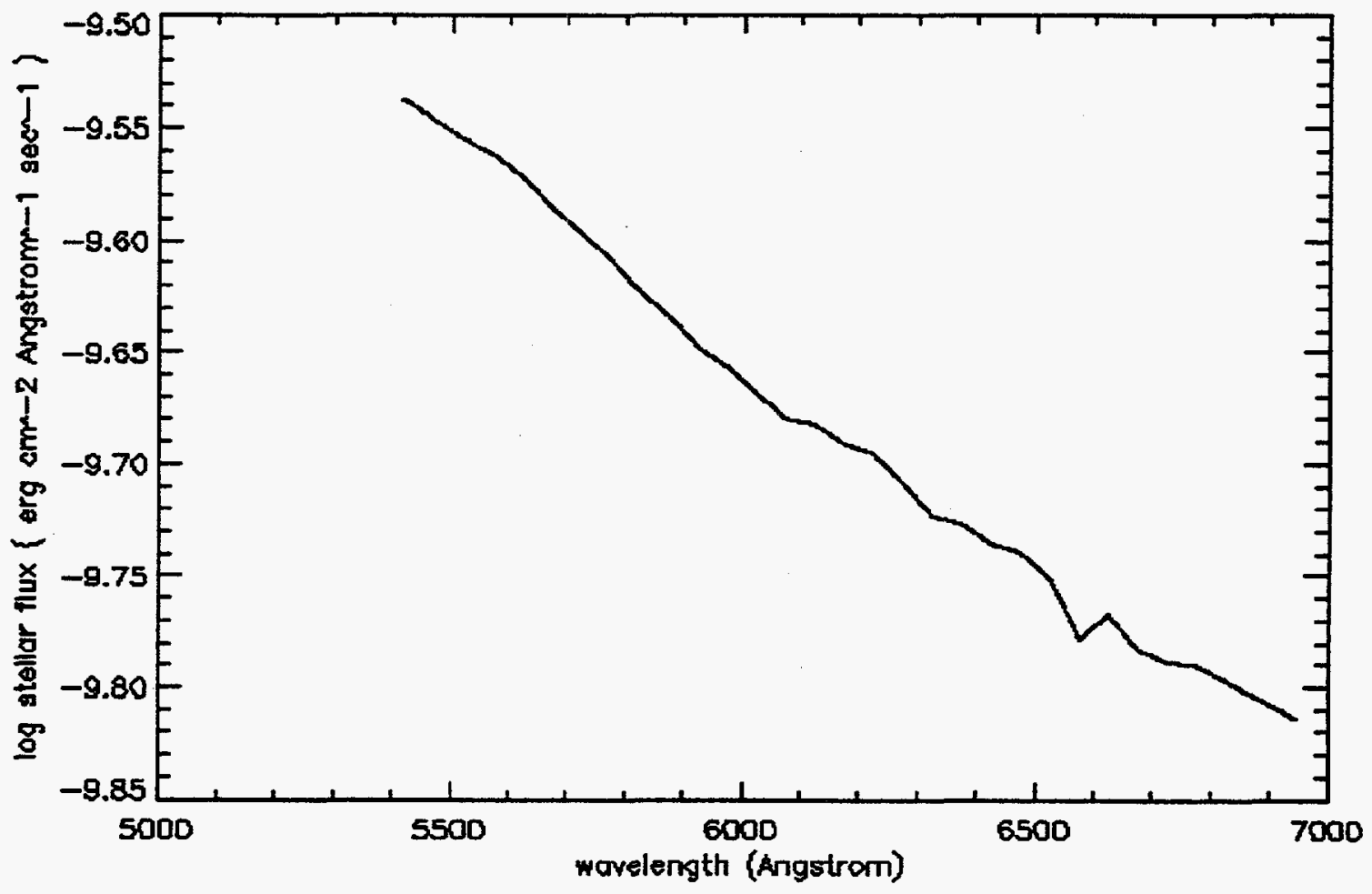

Figure 6 : Extra-terrestrial flux of Zeta Persei. 


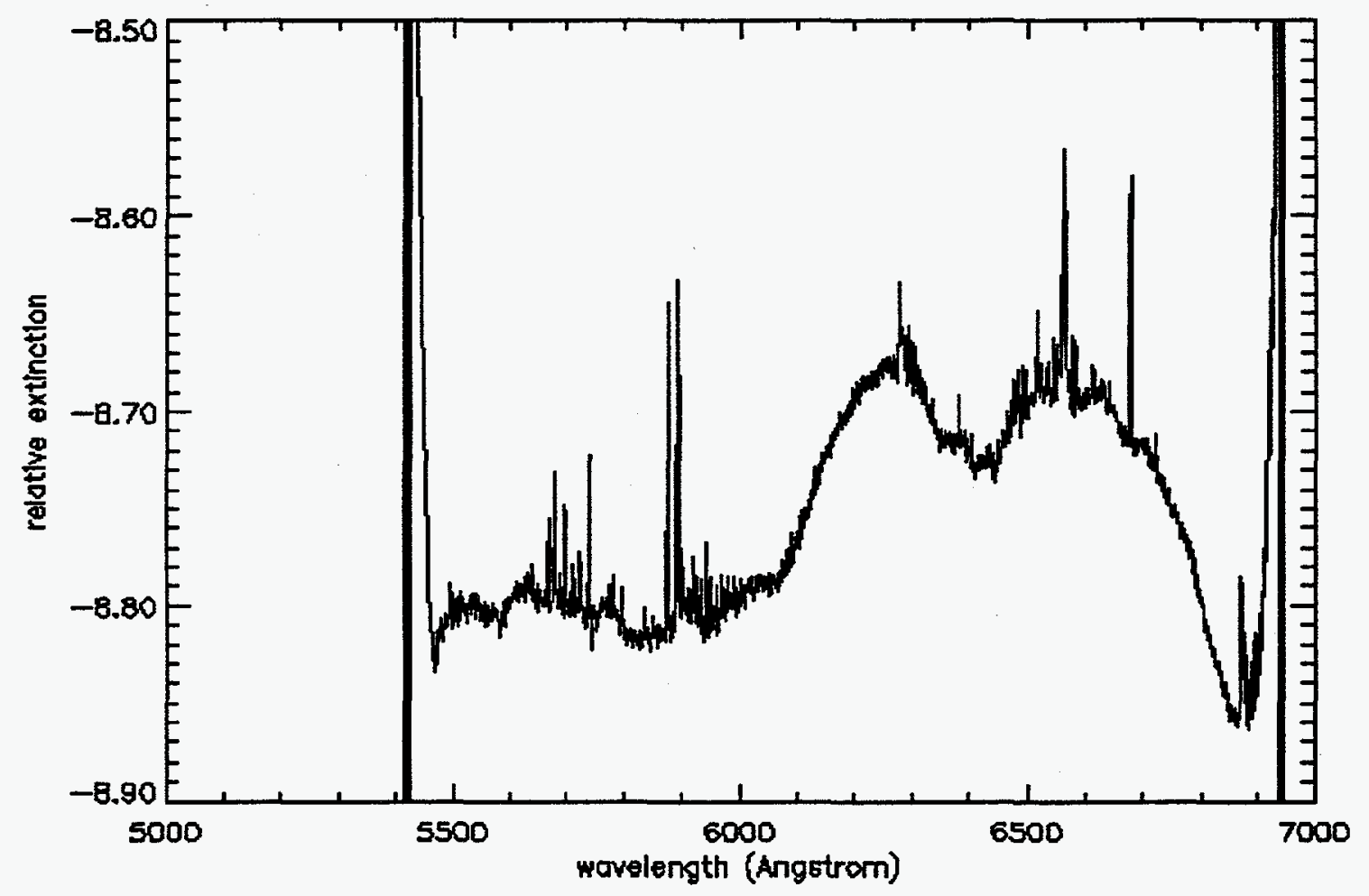

Figure 7 : Relative total extinction. 


\section{APPENDIX}

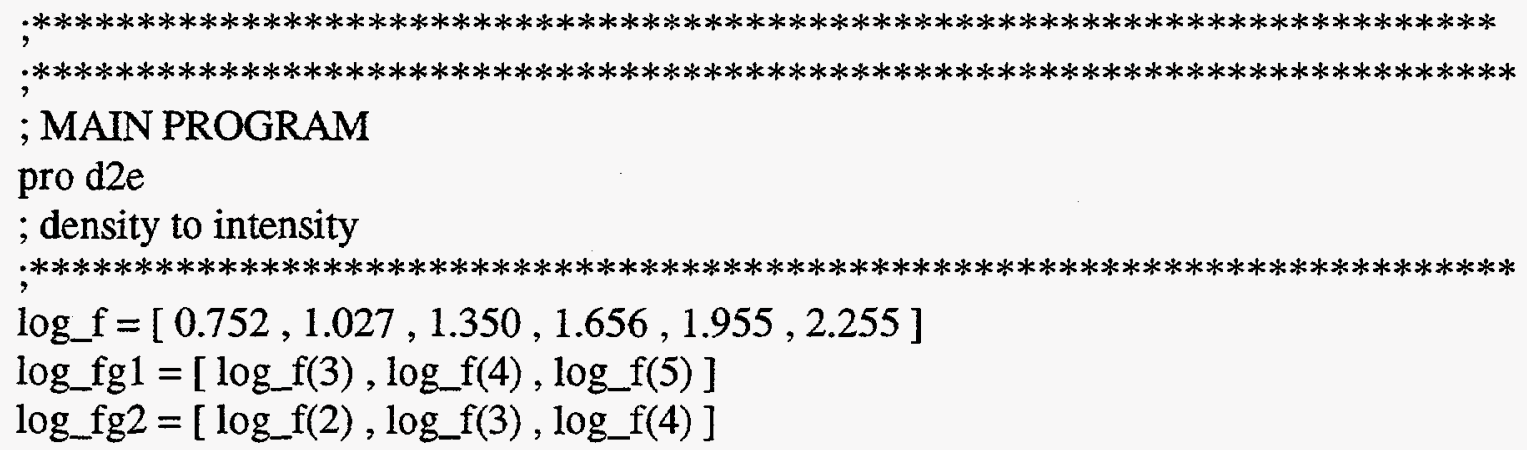


glush = zeta*1e-8; $\mathrm{ergs} /\left(\mathrm{cm}^{\wedge} 2\right.$ Angstrom $\left.\mathrm{sec}\right)$

;***************************SENSITIVITY**********************************************

sense $=$ fltarr $(2,29)$

free_lun, 4

openr, 4, './input/103a-f2.dat'

readf, 4 , sense

free_lun, 4

sensitivity $=\operatorname{smooth}\left(\right.$ interpolate $\left(\right.$ reform $\left(\operatorname{sense}\left(1,{ }^{*}\right)\right),($ wave -5000$\left.\left.) * 7 / 500\right) * 2 / 99,87\right)$

$* * * * * * * * * * * * * * * * * * * *$ CHAPPUIS BAND CM^ 1 1 ${ }^{* * * * * * * * * * * * * * * * * * * * * * * * * * * * * * *}$

chap $=$ fltarr $(2,43)$

free_lun, 5

openr, 5, './input/chap_band.dat'

readf, 5 , chap

free_lun, 5

$\max =\operatorname{chap}(0,42)-\operatorname{chap}(0,0)$

chappuis $=$ fltarr $(1, \max +1)$

lambda $=$ fltarr $(1, \max +1)$

slope $=$ fltarr $(1,42)$

for $\mathrm{i}=0,41$ do begin

endfor

$$
\text { slope }(\mathbf{i})=(\operatorname{chap}(1, \mathbf{i}+1)-\operatorname{chap}(1, \mathbf{i})) /(\operatorname{chap}(0, \mathbf{i}+1)-\operatorname{chap}(0, \mathbf{i}))
$$

$\mathrm{j}=0$

for $\mathrm{i}=0$, max do begin

lambda $(i)=\operatorname{chap}(0,0)+i$

if ( lambda(i) gt chap $(0, j+1)$ ) then $j=j+1$

chappuis $(\mathbf{i})=\operatorname{slope}(\mathbf{j}) *(\operatorname{lambda}(\mathrm{i})-\operatorname{chap}(0, \mathrm{j}))+\operatorname{chap}(1, \mathrm{j})$

endfor

chapp_interp $=$ interpolate $($ reform(chappuis), wave-chap $(0,0)$ )

;***********************CALCULATION*********************************************

gammma $=$ fltarr $(1,2687)$

log_absorp_J_I $=$ fltarr $(1,2687)$

level_intercept $=$ fitarr $(1,2687)$

count $=$ indgen $(2687)$

for $\mathrm{i}=0,2686$ do begin

density $1=[\operatorname{slit} 4(\mathbf{i}), \operatorname{slit5}(\mathbf{i}), \operatorname{slit6(i)}]$

density2 $=[$ slit3(i), slit4(i), slit5(i) $]$

recipro $=\operatorname{maxtr}($ density $1, \operatorname{star}(1, \mathbf{i}), \log$ ffg 1$)$

gammma $(\mathbf{i})=\operatorname{recipro}(0)$

level_intercept $(\mathrm{i})=$ recipro $(1)$

if ( recipro(2) it $\log _{\_} f(3)$ ) then $\$$

endfor

$$
\text { recipro }=\operatorname{maxtr}\left(\text { density } 2, \operatorname{star}(1, \mathbf{i}), \log \_ \text {fg } 2\right)
$$

log_absorp_J $=(\operatorname{star}(1, *)-0.6) /$ gammma - sensitivity 
;***********************WRITE DATA********************************************** absorption $=$ fltarr $(6,2687)$

absorption $\left(0,{ }^{*}\right)=$ count

absorption $(1, *)=$ wave

absorption $\left(2,{ }^{*}\right)=\operatorname{alog} 10$ (glush ) - log_absorp_J

absorption $\left(3,{ }^{*}\right)=$ chapp_interp

absorption $(4, *)=\operatorname{star}(1, *)$

absorption $(5, *)=$ gammma

free_lun, 6

openw, 6, './input/absorption.dat'

printf, 6 , absorption

free_lun, 6

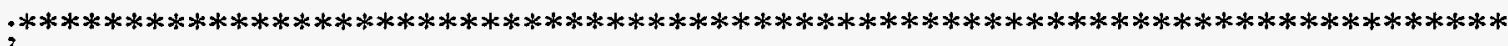
end

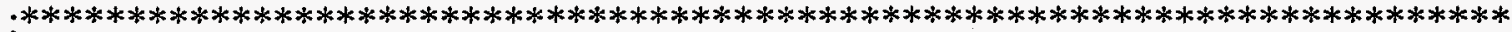
*************************************************************************************** 
$* * * * * * * * * * * * * * * * * * * * * * * * * * * * * * * * * * * * * * * * * * * * * * * * * * * * * * * * * * * * * * * * * * * * * * * * * * * * * * * * * *$

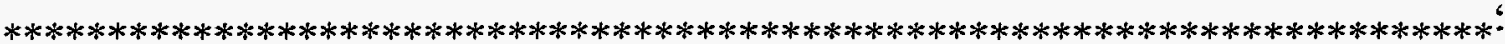

pù

[ 1 $* * * * * * * * * * * * * * * * * * * * * * * * * * * * * * * * * * * * * * * * * * * * * * * * * * * * * * * * * * * * * * * * * * * * * * * * * *$

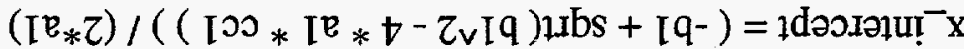

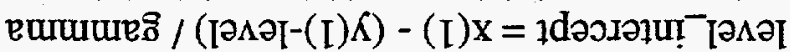
$[\mathrm{q}+Z /((\mathrm{Z}) \mathrm{x}+(\mathrm{I}) \mathrm{x}) *[\mathrm{E} * Z=$ puidures $K \Lambda-[0=[00$

(乙) $[$ Iоџอว $\Lambda=[0$

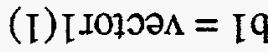

(0) $[$ IOTOOA $=[\mathrm{T}$

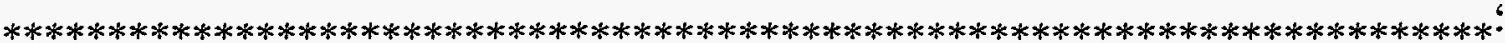

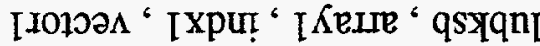
Ip " I Xpu! " [ KeILe " duopnT

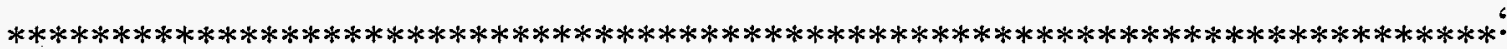

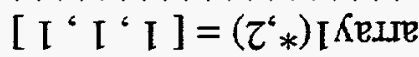

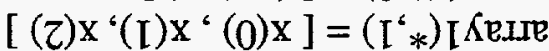

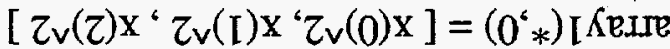
$\left[(\mathrm{Z}) K^{\circ}(\mathrm{I}) K^{\circ}(0) K\right]=[$ IO10วA

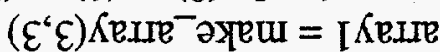

$$
9^{\circ} 0=[\partial \Lambda \partial]
$$

$* * * * * * * * * * * * * * * * * * * * * * * * * * * * * * * * * * * * * * * * * * * * * * * * * * * * * * * * * * * * * * * * * * * * * * * * * * *$

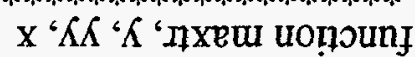

NOLIDNกA NOILVTOddG.LNI LNIOd GGYHI $* * * * * * * * * * * * * * * * * * * * * * * * * * * * * * * * * * * * * * * * * * * * * * * * * * * * * * * * * * * * * * * * * * * * * * * * * * * * * * * * * * *$ $* * * * * * * * * * * * * * * * * * * * * * * * * * * * * * * * * * * * * * * * * * * * * * * * * * * * * * * * * * * * * * * * * * * * * * * * * * *$ 


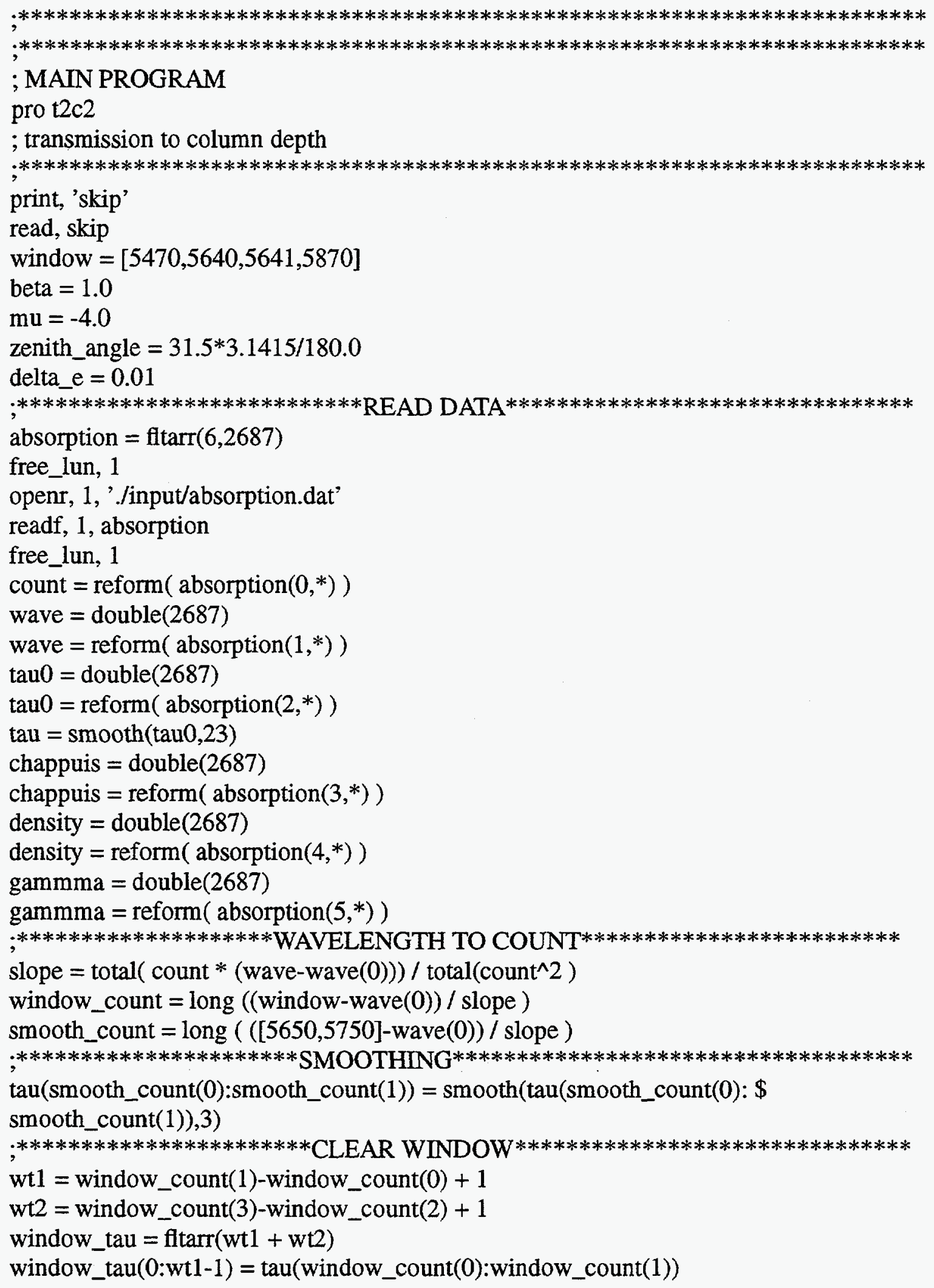


window_tau(wt1:wt $1+w t 2-1)=\operatorname{tau}($ window_count(2):window_count(3))

window_wave $=$ fltarr $(w t 1+w t 2)$

window_wave $(0:$ wt1-1) $=$ wave $($ window_count $(0)$ : window_count $(1))$

window_wave $($ wt 1 :wt $1+w t 2-1)=$ wave $($ window_count(2):window_count $(3))$

window_chap $=$ fltarr $(w t 1+w t 2)$

window_chap $(0:$ wt $1-1)=$ chappuis $($ window_count $(0)$ :window_count $(1))$

window_chap $(w t 1: w t 1+w t 2-1)=$ chappuis $($ window_count(2):window_count(3))

window_density $=$ ftarr $(w t 1+w t 2)$

window_density $(0: w t 1-1)=\operatorname{density}($ window_count $(0)$ :window_count $(1))$

window_density(wt1:wt1+wt2-1) $=$ density (window_count(2):window_count(3))

window_gammma $=$ fltarr $(w t 1+w t 2)$

window_gammma $(0:$ wt $1-1)=$ gammma $($ window_count $(0)$ :window_count $(1))$

window_gammma (wt1:wt1+wt2-1) $=$ gammma (window_count(2):window_count(3))

;*******************************)DIFFERENTIAL $* * * * * * * * * * * * * * * * * * * * * * * * * * * * * * * * * * *$

d_tau $=$ fitarr $(32000)$

d_chap $=$ fltarr $(32000)$

d_wave $=$ fltarr $(32000)$

d_aero $=$ fltarr $(32000)$

d_ray $=$ fitarr $(32000)$

$i=0$

p_min $=0$

p_max $=w t 1+w t 2-1$

S_max $=$ p_max

for $\mathrm{j}=\mathrm{p} \_$min, $p_{-}$max $/ 2$, skip do begin

$\mathrm{k}=\mathrm{j}+\left(\left(p_{-} \_\max -p_{-}\right.\right.$min $\left.) / 2\right)$

d_tau(i) = window_tau(k) - window_tau(j)

d_chap $(\mathrm{i})=$ window_chap $(\mathrm{k})$ - window_chap $(\mathrm{j})$

d_aero(i) $=$ window_wave $(k)^{\wedge}$ beta - window_wave $(j)^{\wedge}$ beta

d_ray $(\mathrm{i})=$ window_wave $(\mathrm{k})^{\wedge} \mathrm{mu}-$ window_wave $(\mathrm{j})^{\wedge} \mathrm{mu}$

$i=i+1$

endfor

numb $=\mathrm{i}$

;*************************** LEAST SQUARES************************************

$\mathrm{Y} 1=\operatorname{total}\left(\mathrm{d} \_\right.$tau $*$ d_ray)

$\mathrm{Y} 2=\operatorname{total}($ d_tau*d_aero)

$\mathrm{Y} 3=$ total(d_tau*d_chap)

$\mathrm{Al}=\operatorname{total}($ d_ray 2 )

A2 $=$ total(d_ray*d_aero)

$\mathrm{A} 3=$ total(d_ray*d_chap)

$\mathrm{B} 1=$ total(d_aero*d_ray)

$\mathrm{B} 2=$ total $\left(\right.$ d_aero $\left.^{\wedge} 2\right)$

B3 $=$ total(d_aero $*$ d_chap)

$\mathrm{C} 1=$ total(d_chap*d_ray) 
$\mathrm{C} 2$ = total(d_chap*d_aero)

C3 = total(d_chap $\left.{ }^{\wedge} 2\right)$

vector $=[\mathrm{Y} 1, \mathrm{Y} 2, \mathrm{Y} 3]$

array $=$ make_array $(3,3)$

$\operatorname{array}(0, *)=[\mathrm{A} 1, \mathrm{~B} 1, \mathrm{C} 1]$

$\operatorname{array}(1, *)=[\mathrm{A} 2, \mathrm{~B} 2, \mathrm{C} 2]$

$\operatorname{array}\left(2,{ }^{*}\right)=[\mathrm{A} 3, \mathrm{~B} 3, \mathrm{C} 3]$

ludcmp, array, indx $1, \mathrm{~d} 1$

lubksb, array, indx1, vector

rho $=$ vector $(0)$

alpha $=$ vector $(1)$

ada $=\operatorname{vector}(2)$

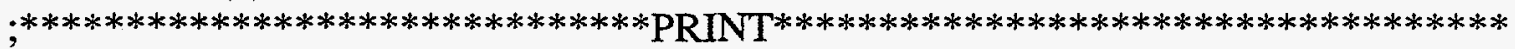

print, 'skip = ", skip

print, "sensitivity uncertainty $=$ ", delta_e

print, "points $=$ ", wt $1+w t 2$

print, "rho $=$ ", rho

print, 'alpha = ", alpha

print, 'ada = ', ada

print, 'column = ', ada*cos(zenith_angle)

print, 'combinations $=$ ', numb

print, 'ada uncertainty $=$ ', delta_e* $\operatorname{sqrt}(2) / \operatorname{sqrt}\left(\operatorname{total}\left(d_{-}\right.\right.$chap^$\left.^{\wedge} 2\right)$ )

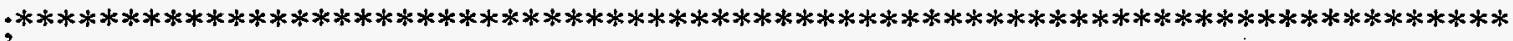

end

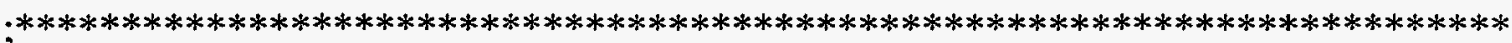

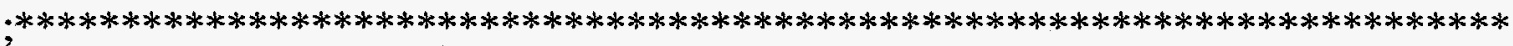




\section{BIBLIOGRAPHY}

Aller, L.H., Astrophysics: The Atmospheres of the Sun and Stars, (New York : The Ronald Press Company, 1963).

Angione, R.J., Medeiros, E.J. and R.G. Rosen, "Stratospheric ozone as viewed from Chappuis band", Nature, 261, pp. 289-290, (1976).

Angione, R.J. and R. G.Roosen, "Use of the chappuis band for determination of modern and historical ozone levels", Final Report to NASA/Langley on Grant NSG 1430, (1981).

Angione, R. J. and R. G. Roosen, "Baseline ozone results from 1923 to 1955 ", J. Clim. and Appl. Meteor., 22, pp. 1377-1383, (1983).

Bower, F.A. and R.B. Ward, Stratospheric Ozone and Man, vol. 2, (Boca Raton, FL : CRC Press, 1982).

Brasseur, G. and S. Solomon, Aeronomy of the Middle Atmosphere, (Boston : D. Reidel Publishing Company, 1984).

Buglia, J.J., Introduction to the Theory of Atmospheric Radiative Transfer, (Hampton,VA : NASA, 1986).

Cicerone, R.J., "Changes in stratospheric ozone”, Science, 237, pp. 35-42, (1987).

Dobson, G.M.B. "Forty years' research on atmospheric ozone at Oxford : a history", Applied Optics, 7, pp. 387-405, (1968).

Glushneva, I. N., A. V. Kharitonov, L. N. Knyazeva, and V. I. Shenavrin, "Secondary spectrophotometric standards", $A \& A S S, 92$, pp. 1-29, (1992).

Griggs, M., "Absorption coefficients of ozone in the ultraviolet and visible regions", $J$. Chem. Phys., 49, No. 2, pp. 857-859, (1968).

Grossman, A.S., Grant, K.E., and D.J. Wuebbles, "Radiative flux calculations at UV and visible wavelengths”, LLNL Report UCRL-ID-115336, (1993).

Grossman, A.S., and K.E. Grant, "A correlated k-distribution model of the heating rates for atmospheric mixtures of $\mathrm{H}_{2} \mathrm{O}, \mathrm{CO}_{2}, \mathrm{O}_{3}, \mathrm{CH}_{4}$ and $\mathrm{N}_{2} \mathrm{O}$ in the $0-2500 \mathrm{~cm}^{-1}$ wave number region at altitudes between 0 and $60 \mathrm{~km}$ ", Submitted to the $8^{\text {th }}$ Conference on Atmospheric Radiation, American Meteorological Society, Nashville, Tenn., Jan. 23-28, (1994). 
Grossman, A.S., and K.E. Grant, "Effects of tropospheric aerosols on UV and visible surface fluxes", LLNL Report UCRL-JC-118055. Submitted to the 1994 Fall Meeting of the American Geophysical Union, San Francisco, Calif., (1994).

Grossman, A.S., "Proposal to NASA: Terrestrial ozone abundance determinations using photographic stellar spectra measurements”, (1995).

Hamill, P. and O.B. Toon, "Polar stratospheric clouds and the ozone hole", Physics Today, pp. 34-42, (1991).

Herbig, G.H., "The diffuse interstellar bands. IV. The region 4400-6850 A", Ap. J., 196, pp. 129-160, (1975).

Houghton, H.G., Physical Meteorology, (Cambridge, MA : The MIT Press, 1985).

Humphrey, G.L. and R.M. Badger, "The absorption spectrum of ozone in the visible", $J$. Chem. Phys., 15, No. 11, pp. 794-798, (1947).

Inn, E.C.Y. and Y. Tanaka, "Absorption coefficient of ozone in the ultraviolet and visible regions”, J. Opt. Soc. Amer, 43, p. 870, (1953).

Intergovernmental Panel on Climate Change, Climate Change; The IPCC Scientific Assessment, (Cambridge, UK : Cambridge University Press, 1990).

Intergovernmental Panel on Climate Change, Climate Change 1992; The Supplementary Report to the IPCC Scientific Assessment, (Cambridge, UK : Cambridge University Press, 1992).

Intergovernmental Panel on Climate Change, Climate Change; The IPCC Scientific Assessment, (Cambridge, UK : Cambridge Unviersity Press, 1994).

James, T.H., Theory of the Photographic Process, (New York : Macmillan, 1977).

Johnson, J.C., Physical Meteorology, (New York : John Wiley \& Sons, 1954).

King, M.D., and D.M. Byrne, "A method for inferring total ozone content from the spectral variation of total optical depth obatined with a solar radiometer", J. Atmos. Sci., 33, pp. 2242-2251, (1976).

Kodak, Technical information of photographic plates presently offered by Kodak 1994, extracted from publication P-315d. 
Kyle,T. G., Atmospheric Transmission, Emission and Scattering, (New York : Pergamon 1991).

Lacis, A.A. and J.E. Hansen, "A parameterization for the absorption of solar radiation in the earth's atmosphere", J. Atmos. Sci., 31, pp. 118-133, (1974).

Levi, L., Applied Optics: A Guide to Optical System Design, vol. 2, (New York : John Wiley \& Sons, 1980).

Mehra, P. , R. Vijayakumar and A.M. Selvam, "Measurement of atmospheric total ozone by the filter photometric method", J. Atmos. Chem., 4, pp. 335-342, (1986).

Moore, C., M.C. J. Minnaaert and J. Houtgast, "The solar spectrum 2935 A to 8770 A", National Bureau of Standards Monograph, Number 61, (1966).

Morton, R. A., Photography for the Scientist, (Orlando: Academic, 1984).

National Research Council, Causes and Effects of Stratospheric Ozone Reduction: An Update, (Washington, DC : National Academy Press, 1982).

Rindert, S.B., "Atmospheric Ozone at Uppsala, Sweden, 1951-1966", Department of Meterology Report No. 45 (Uppsala, Landstingets Verkstader), (1976).

Schachter, J., "Ozone Absorption Bands in the 3100A-3400A Region", PASP, 103, pp. 457-460, (1991).

Shaw, G., "Atmospheric ozone : determination by Chappuis band absorption", J. Appl. Meteor., 18, pp. 1335-1339, (1979).

Soderblom, D.R., "Coude Spectroscopy at Lick Observatory", Lick Observatory Technical Reports, No. 18 , (1976).

Storer, N.W., "A photometric study of the continuous spectra of giant and dwarf stars", Lick Observatory Bulletin, XIV , No. 410, pp. 41-57, (1928).

Theodossiou, E., "Empirical effective temperatures of late O, B, A and early F stars", Mon. Not. R. astr. Soc., 214, pp. 327-335, (1985).

Turco, R.P., Toon, O.B., Park, C., Whitten, R.C. and J. B. Pollack, and P. Noerdlinger, "Tunguska meteor fall of 1908 : effects on stratospheric ozone", Science, 214, pp. 19-23, (1981). 
University Corporation for Atmospheric Research and National Oceanic and Atmospheric Adminstration, "Our Ozone Shield", Reports to the Nation On Our Changing Planet, Fall, No. 2, (1992).

UV-B Monitoring Workshop, "A Review of the Science and Status of Measuring and Monitoring Programs", sponsored by the Alternative Fluorocarbons Environmental Acceptability Study, and the Cooperative State Research Service, U.S. Department of Agriculture, Conducted and reported on by Science and Policy Associates Inc., The West Tower, Suite 400, 1333 H Street, Washington D. C., (1992)

Walker, G., Astronomical Observations : An Optical Perspective, (New York : Cambridge University Press, 1992).

Whitten, R.C. and S.S. Prasad, Ozone in the Free Atmosphere, (New York : Van Nostrand Reinhold, 1985).

World Meteorological Organization, "Scientific Assessment of Ozone Depletion: 1991", Global Ozone Research and Monitorning Project Report 25, Geneva, (1991).

World Meteorological Organization, "Scientific Assessment of Ozone Depletion: 1994", Global Ozone Research and Monitorning Project Report 25, Geneva, (1994). 